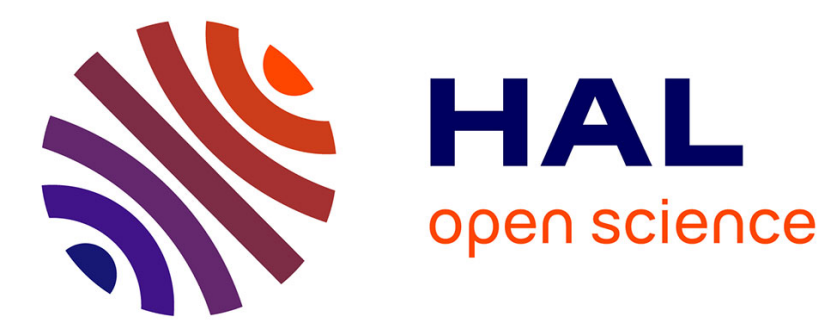

\title{
Changements anthroponymiques et sociogenèse de la noblesse en Franconie à la fin du Moyen Âge \\ Joseph Morsel
}

\section{To cite this version:}

Joseph Morsel. Changements anthroponymiques et sociogenèse de la noblesse en Franconie à la fin du Moyen Âge. Changements anthroponymiques et sociogenèse de la noblesse en Franconie à la fin du Moyen Âge, 1993, Göttingen, Allemagne. pp.89-119. halshs-00289945

\section{HAL Id: halshs-00289945 https://shs.hal.science/halshs-00289945}

Submitted on 24 Jun 2008

HAL is a multi-disciplinary open access archive for the deposit and dissemination of scientific research documents, whether they are published or not. The documents may come from teaching and research institutions in France or abroad, or from public or private research centers.
L'archive ouverte pluridisciplinaire HAL, est destinée au dépôt et à la diffusion de documents scientifiques de niveau recherche, publiés ou non, émanant des établissements d'enseignement et de recherche français ou étrangers, des laboratoires publics ou privés. 
Monique Bourin, Pascal Chareille (dir.), Genèse médiévale de l'anthroponymie moderne, III : Enquêtes généalogiques et données prosopographiques, Publications de l’Université de Tours, 1995.

Texte des pages 89-119 et planches hors-texte 17-19

\title{
CHANGEMENTS ANTHROPONYMIQUES ET SOCIOGENÈSE DE LA NOBLESSE EN FRANCONIE À LA FIN DU MOYEN ÂGE*
}

\author{
Joseph MORSEL \\ Mission Historique Française en Allemagne (Göttingen)
}

\begin{abstract}
La femme d'un des sauvages qui m'avaient fait prisonnier ayant mis au monde un enfant, au bout de quelques jours le père convoqua ses voisins dans la cabane pour chercher quel nom on pourrait lui donner. Il en voulait un qui exprimât la vaillance et le rendît redoutable. Ses voisins lui en proposèrent plusieurs mais il ne voulut pas les accepter. Finalement, il déclara qu'il lui donnerait le nom d'un de leurs quatre ancêtres, qui sont: Quirima, Eramittan, Coem, je ne me rappelle pas le quatrième. Je pensai d'abord que Coem était le même que Cham [...] et je lui conseillai de le choisir, car c'aurait été en effet celui d'un de ses ancêtres. On donna un de ces quatre noms à l'enfant; et c'est ainsi qu'ils agissent sans plus de cérémonie.

Hans STADEN, Warhaftige Historia und Beschreibung eyner Landtschafft... in der Newenweit America gelegen..., 1557.
\end{abstract}

Identifiant les personnes, c'est-à-dire en fin de compte construisant les agents sociaux ${ }^{1}$, revient aux anthroponymes le lourd privilège de constituer [9o] (aux deux sens du terme) l'une des dimensions essentielles de la socialisation, c'est-à-dire à la fois de la mise en rapport des personnes et la représentation de cette mise en rapport. C'est ce qui fait l'importance de l'étude des anthroponymes pour les historiens de la société : on peut poser comme postulat de base que les changements observés dans les pratiques anthroponymiques ne sont pas de simples accidents de parcours mais correspondent à des changements profonds affectant les rapports sociaux - le problème étant de savoir de quelle nature est cette correspondance, si celle-ci varie en fonction de la dimension sociale des rapports concernés et si les propriétés sociales de l'écriture n’introduisent pas un filtre spécifique ${ }^{2}$.

Le cas de la petite noblesse franconienne de la fin du Moyen Âge constitue un observatoire intéressant pour ce type de problèmes. D'une part, la Franconie forme, avec d'autres régions méridionales de l'Empire comme la Souabe et le fossé rhénan, un espace où la (petite) noblesse a connu une évolution particulière par rapport aux autres

\footnotetext{
* Une première version de ce texte a été présentée lors du colloque «Nom et changement social/Namen und sozialer Wandel », organisé à Göttingen les 6-7 décembre 1993 par la Mission Historique Française en Allemagne avec la collaboration du GDR 0955, de l'Arbeitsgruppe zur Erforschung der mittelalterlichen Anthroponymie et du MaxPlanck-Institut fur Geschichte. Que tous les participants à la discussion y afférente en soient ici remerciés, ainsi que Dominique Barthélemy, Jean-Louis Biget, Olivier Christin, Egon Flaig, Anita et Alain Guerreau et Martial Staub pour leurs précieuses remarques.

${ }^{1}$ Hegel indique clairement combien le nom est à la fois l'instrument d'identification d'une personne et le corollaire de sa mise en rapport avec les autres, donc à la fois produit et facteur de sa mise en rapport : « C'est le nom et lui seul qui fait que la différence du singulier d'avec tous les autres est non pas signifiée mais bien rendue réelle par tous ceux qui parlent. Dans le nom, le singulier ne vaut plus en tant que ce singulier qu'il est dans sa seule conscience, mais dans la conscience de tous. » (Georg Wilheim Friedrich Hegel, Phénoménologie de l'esprit, (trad. franç. de Jean Hyppolite) Paris : Aubier, 1947, t. 2, p. 72). Voir aussi le commentaire de Vincent DescombES, L’inconscient malgré lui, Paris : Minuit, 1977, p. 47: «La nomination ne consiste pas à trouver un mot pour quelqu'un qui serait déjà là [...], l'être unique de celui qui est seul à être celui qu'il est. Elle fait de la différence [Derrida dirait : la différance] de l'un et de tous les autres une différence réelle », première exigence à la formation d'un rapport social, mais exigence impensable hors de cette formation d'un rapport social. La « différence réelle » n'a pas d'existence autonome, elle n'est produite que dans le cadre d'un rapport social. À cet égard, il est bon d'insister sur le fait que les anthroponymes étudiés par les historiens sont des anthroponymes utilisés (et non pas virtuels), donc que ceux-ci ne sont pas de simples identifiants de personnes, mais des modes d'inscription de ces personnes dans des rapports sociaux dont les textes ne sont pas simplement la mise en mémoire mais la réalisation.

${ }^{2}$ Sur le problème de la scripturalité (terme que je propose comme équivalent à l'anglais literacy et à l'allemand Schriftlichkeit), je me permets de renvoyer à ma thèse : Une société politique en Franconie à la fin du Moyen Age : les Thüngen, leurs princes, leurs pairs et leurs hommes (1275-1525), Paris-IV : ms. dactylogr., 1993,1 partie.
} 
aristocraties de l'Empire et même de l'Occident, évolution par laquelle elle a échappé au changement social accompagnant ailleurs la genèse de l'Etat moderne's, pour former au XVI ${ }^{\mathrm{e}}$ siècle la Chevalerie d'Empire (Reichsritterschaft). D'autre part, on dispose d'un corpus anthroponymique assez considérable, fruit à la fois de la relative richesse des sources par rapport à d'autres régions de l'Empire et de l'existence d'instruments de travail permettant l'extraction assez aisée des anthroponymes nobiliaires4.

[91] Enfin, l'étude de ces anthroponymes permet d'observer quelques mutations notables des pratiques anthroponymiques dans la noblesse de la fin du Moyen Âge, qu'il apparaît non seulement intéressant mais surtout indispensable de corréler avec les changements qui affectent les rapports sociaux dans lesquels sont impliqués les nobles franconiens. Les rapports sociaux ne fonctionnent en effet pas sans être construits idéellement, mettant ainsi les hommes en rapport et en même temps affectant cette mise en rapport d'une valeur sociale ${ }^{5}$. Et dans la mesure où la construction idéelle (consciente et inconsciente) passe prioritairement par le langage, on comprend à la fois l'importance de la formalisation langagière dans la formation du monde social et, consécutivement, l'intérêt qui doit être porté aux actes de langage et à l'ensemble des procédures de nomination par lesquels les agents sociaux, les positions sociales et les rapports sociaux sont construits et reconstruits.

\section{Vicissitudes patronymiques}

En premier lieu (sans préjuger de l'importance ni même de la situation temporelle, mais en se basant sur la visibilité du phénomène), une mutation complexe affecte les pratiques patronymiques nobiliaires.

\section{a. Patronymes féminins : l'invention du père}

Lorsque l'on observe les tableaux généalogiques établis pour les petits nobles ${ }^{6}$, on remarque la large méconnaissance du patronyme (nomen paternum) des épouses du $\mathrm{XIV}^{\mathrm{e}}$ siècle, dont on ne connaît bien souvent que le prénom, contrairement à ce qui se passe pour le $\mathrm{XV}$ e siècle : le problème est ici donc moins - contrairement aux siècles

\footnotetext{
3 Sur la spécificité du développement social de la noblesse franconienne à la fin du Moyen Âge, je me permets de renvoyer encore une fois à ma thèse citée à la note précédente, ainsi qu'à ma contribution « Die Erfindung des Adels. Zur Soziogenese des Adels am Ende des Mittelalters am Beispiel Frankens » au colloque Nobilitas. Funktion und Repräsentation des Adels in Alteuropa (Schloß Ringberg über dem Tegernsee, 20-23 Februar 1994), à paraître à Göttingen (Vandenhoeck \& Ruprecht) en 1995.

4 Comme dans les autres régions méridionales citées, on dispose de diverses listes de nobles établies à l'occasion des grands tournois nobiliaires de la fin du Moyen Âge ou d'événements comme les noces du duc de Bavière-Landshut (Hans SEIBOLD, "Gleichzeitige und vollständige Beschreibung der berühmten Hochzeit H. Georg des Reichen zu Landshut 1475 », in : Lorenz WESTENRIEDER (éd.), Beyträge zur vaterländischen Historie, Geographie, Statistik und Landwirtschaft, t. 2, München, 1789, p. 105-221). Toutefois, la Franconie bénéficie d'autres avantages archivistiques et instrumentaux, comme la série des livres de fiefs des évêques de Wurtzbourg commencée dès 1303 et exploitée par informatique, tout comme le plus ancien censier épiscopal (1475) (Bayerisches Staatsarchiv Würzburg, Lehenbücher, $\mathrm{Nr} 5,11,21,30,31$ et Salbücher, $\mathrm{Nr} 1$; exploitation informatique présentée par Rolf SPRANDEL et Hans-Peter BAUM, « Die Erforschung von Lehensregistern in Verbindung mit der EDV. Ein sozialgeschichtliches Projekt der Universität Würzburg ", Jahrbuch der historischen Forschung, 1980, p. 49-55), les listes éditées des nobles alliés au margrave de Brandebourg-Ansbach contre la ville de Nuremberg en 1449-1450 (Joseph BADER (éd.), Erhard Schürstab's Beschreibung des Ersten Markgraflichen Krieges gegen Nürnberg, München, 186o, Beil. 1 et note 1; K. HEGEL (éd.), Chroniken der fränkischen Städte - Nürnberg, t. 2, Leipzig, 1862, V, Beil. 2) ou encore les listes nominatives établies en 1526 pour l'indemnisation des nobles victimes de la Guerre des Paysans (Bayerisches Staatsarchiv Würzburg, Miscellanea, $\mathrm{Nr} 484$ et 1066/I-II).

5 Cette valeur peut être positive (légitimant le rapport et incitant à le reproduire) ou négative (condamnant un rapport, éventuellement mythique, et incitant à le détruire ou, s’il est mythique, à empêcher son avènement éventuellement présenté comme un retour au chaos). Sur ces deux dimensions du travail social (travail de regroupement, travail de valorisation/délimitation), voir Luc BOLTANSKI, Les cadres. La formation d'un groupe social, Paris : Minuit, 1982, notamment p. 51-59; Maurice GodELIER, L’idéel et le matériel. Pensée, économies, sociétés, Paris : Fayard, 1984, notamment p. 221-228; et aussi Alain DESROSIÈRES, La politique des grands nombres. Histoire de la raison statistique, Paris : La Découverte, 1993, notamment p. 7-27.

6 Par exemple Walther MölleR, Stamm-Tafeln westdeutscher Adels-Geschlechter im Mittelalter, N.F., t 2, Darmstadt,
} 1951. 
précédents - de sous-représentation féminine (toutefois encore nette) que de "sousidentification ». La raison en est simple : les documents (textes, sceaux, inscriptions monumentales) confèrent [92] généralement aux épouses le patronyme et les armoiries de leur époux, lorsqu'ils ne se contentent pas de mentionner leur prénom en précisant, selon les cas, "épouse de » ou "veuve de " tel homme. Le caractère tendanciellement taisible de l'identité de naissance de la femme est parfois contrecarré par des cas de mention expresse, qui ont implicitement le mérite de montrer, par leur existence même et leur logique propre, que ce caractère taisible était la règle : l'inverse était pensable, mais implicitement réservé à certains enjeux, à savoir l'hypergamie masculine, dans laquelle les intérêts patrimoniaux (dont la préservation est l'objet prioritairement des unions isogamiques voire hypogamiques) se doublent (lorsqu'ils ne restent pas dans l'ombre) d'intérêts symboliques (le prestige attaché à l'alliance avec une parentèle prestigieuse)7. Dans le cas «normal», les femmes ne voient généralement leur identité signalée ni par les lignées donatrices, ni par les lignées donataires. Mais comme il est très peu probable que cela signifie que les objets échangés (les femmes) n'avaient aucune valeur sociale, ou que l'échange n'avait aucune importance, il faut certainement interpréter ce mutisme plutôt comme le résultat d'une absence de nécessité sociale, l'écriture et l'héraldique n'étant pas chargées de livrer à l'éternité et/ou ailleurs le souvenir et/ou la mention de cette alliance matrimoniale spécifique.

Ceci donne toute son importance au changement observable à partir de la fin du XIVe siècle. Du point de vue héraldique, on voit apparaître pour les dames de petite noblesse un nouveau type (déjà connu en revanche dans la haute aristocratie ${ }^{8}$, où les enjeux matrimoniaux plus vastes que pour la petite noblesse, à l'image de l'extension spatiale du réseau matrimonial, confèrent sans doute aux femmes une valeur sociale plus grande - et donc un mode d'identification plus prégnant), celui de la « femmepont », brandissant dans une main l'écu de son père et dans l'autre celui de son époux, la légende continuant toutefois de substituer le patronyme du mari à celui du père. Le sceau restitue ainsi sous la forme iconique d'une double appartenance lignagère (par naissance/par mariage) la double appartenance parentale [93] (consanguinité/affinité) de la femme et par là l'alliance de deux parentèles ${ }^{9}$ réalisée par le mariage.

\footnotetext{
7 Une situation héraldique semblable paraît pouvoir être observée pour les femmes de haute noblesse en Rhénanie et Westphalie du XIII siècle, où la seule femme qui fait figurer sur son sceau le seul écu de son père est précisément de naissance supérieure (comtale) à celle de son époux : voir Andrea STIELDORF, Erste Zeugnisse für Besiegelung durch Frauen in rheinischen und westfälischen Territorien, Bonn : ms. dactyl. (Staatsexamsarbeit), 1992, p. 114.

8 Ce type se rencontre dès le XIII ${ }^{\text {e }}$. chez les dames de haute noblesse de Rhénanie et Westphalie (Andrea STIELDORF, Erste Zeugnisse..., p. 114-115), et Michel PASTOUREAU, Traité d'héraldique, ${ }^{\mathrm{e}}{ }^{\mathrm{e}}$ d. Paris : Picard, 1993, p. 46-47, en présente quelques exemples du XIVe siècle français concernant tous la haute noblesse. C'est à ce type que se rattache également le sceau de justice (scel aux causes, souverainetés et appelations pour le roi) que s'est fait faire Isabeau de Bavière et que décrit minutieusement Enguerrand de Monstrelet, Chronique (éd. Louis DrouËT D’ARCQ), t. 3, Paris : Société de l'Histoire de France, 1859, p. 234.

9 Une grande confusion règne souvent dans le langage des médiévistes lorsqu'ils traitent de la parenté, fruit d'un emploi non critique de termes imprécisés, issus le plus souvent du langage commun, qu'il soit actuel (par exemple " famille ») ou médiéval (par exemple «lignage »), où ils ont eux-mêmes un sens fort large; la confusion peut d'ailleurs être accrue par l'usage de termes formalisés par une science dont les historiens ignorent les procédures, introduisant ainsi (dans le cas par exemple du "lignage » des anthropologues) une impression trompeuse de similitude lexicale et de clarté sémantique. Pour tenter de clarifier mon propos, je propose de définir ici (en m'appuyant sur les travaux essentiels de Jack Goody, L'évolution de la famille et du mariage en Europe, Paris : Colin, 1985, notamment p. 225241, et d'Anita GUERREAU-JALABERT, qui a dernièrement fourni un remarquable état de la question dans sa contribution «Prohibitions canoniques et stratégies matrimoniales dans l'aristocratie médiévale de la France du Nord », in : Pierre BONTE (dir.), Épouser au plus proche. Inceste, prohibitions et stratégies matrimoniales autour de la Méditerranée, Paris : EHESS, 1994, p. 293-321) les termes suivants : la "parenté » est l'ensemble socialement défini et construit des relations de parenté (par consanguinité, affinité ou parenté spirituelle) constituant un système propre à chaque société. La "parentèle » est un ensemble cognatique d'individus reconnus/se reconnaissant comme parents, qu'il soit polarisé sur un individu (la parentèle d'Ego) ou issu d'un individu (parentèle descendante = l'ensemble des parents se reconnaissant au moins un ancêtre commun). À l'époque considérée (fin du Moyen Âge), les patronymes identifient des "lignées », c'est-à-dire des groupes de filiation ancrés sur au moins un château et/ou une seigneurie (d'où la forme principalement toponymique des patronymes) dont la transmission aux enfants constitue l'une des principales préoccupations des détenteurs de la terre ; lorsqu'il existe plusieurs châteaux et/ou seigneuries, ces lignées peuvent se subdiviser en branches ancrées chacune sur un château (on observe toutefois un entretien
} 
Parallèlement (ou plutôt par la suite ?10) se répandent à partir de la fin du XIVe siècle, pour finir par dominer au XVe siècle, les sceaux féminins ne présentant plus que le patronyme et l'écu paternels, ne se distinguant plus des sceaux de " pucelles ». Les femmes ont donc réintégré sigillographiquement [94] (c'est-à-dire héraldiquement et patronymiquement) leur lignage de naissance, dont elles sont présentées comme un membre à part entière indépendamment de leur état matrimonial éventuel. Ce sont désormais les textes qui se chargent de mentionner la double appartenance lignagère de la femme, à l'aide d'un système anthroponymique triple conçu sur le modèle «[prénom] [patronyme du mari], née [patronyme du père]» (par exemple Margaretha Truchse $\beta$, Steinrückin geboren ${ }^{11}$ ). Ce système patronymique a connu un très grand succès puisqu'il a été utilisé par la Chevalerie d'Empire jusqu'à la fin de l'époque moderne.

On se trouve donc en présence de deux pratiques anthroponymiques, l'une sigillographique, l'autre textuelle, différentes mais non pas opposées : toutes les deux convergent vers l'indication du lignage de naissance de la femme, mais l'une semble ${ }^{12}$ privilégier le lignage de naissance tandis que l'autre insiste sur la double appartenance lignagère de la femme. Il semble d'ailleurs que le sceau ait été (ou fini par être ?) conçu précisément comme un "signe de naissance », si l'on en croit la qualification de leur sceau comme angeborenes Insiegel («sceau inné ») par les sigillants, de plus en plus fréquente dans la seconde moitié du XV siècle. La concentration sur le lignage paternel observable sur nombre de sceaux est vraisemblablement à l'origine de l'innéité prêtée au sceau, puisqu'une causalité inverse aurait interdit le mutisme des sceaux du XIVe siècle quant au lignage de naissance de l'épouse.

Quoi qu'il en fût, il est clair que depuis les alentours de 1400, les femmes sont conçues comme les traces visibles, montrables et mémorables des alliances matrimoniales conclues entre deux topolignées (conçues comme des lignages). Le changement est d'importance: l'échange généralisé des femmes existait déjà antérieurement au niveau pratique (encouragé en cela par les prohibitions canoniques), sans que cela apparaisse nécessairement au niveau scriptural (hormis dans la haute aristocratie et dans les cas d'hypergamie masculine). Désormais, cet échange généralisé apparaît au niveau scriptural : les textes signalent et éternisent les liens d'alliance entre les topolignées.

régulier des relations de parenté, notamment par des bouclages consanguins et des pratiques successorales indivisions ou transmission agnatique des fiefs nobiliaires entre les branches issues d'une même lignée - identifiée, rappelons-le, par un patronyme permettant une éventuelle coalescence des biens au sein de la lignée en question); ces lignées ou branches cristallisées autour de terres précises, différenciées à l'aide de la particule zu suivant le patronyme et suivie du nom du château (éventuellement identique au patronyme), peuvent donc être nommées "lignées possessionnées », « lignées patrimoniales » (la transmission de la terre s'effectuant préférentiellement de père en fils) ou « topolignées » (sur ces différents termes, voir dernièrement A. GUERREAU-JALABERT, op. cit., p. 313-314, à qui j'emprunte le terme de «topolignée »). Le « lignage » (en tant que traduction du terme Geschlecht : dans la France médiévale, le terme de lignage correspond à ce que j'ai défini plus haut comme " parentèle »), enfin, n'est qu'une forme de représentation de la lignée (i.e. une topolignée ou un ensemble de topolignées), sous la forme discursive d'un groupe de filiation patrilinéaire cristallisé autour d'un nom, d'armoiries et d'un cimier (d'où une désignation de plus en plus redondante aboutissant au XVe siècle à l'expression das Geschlecht, Name, Schild und Helm der [von] N* ( "le lignage, nom, écu et cimier des [patronyme avec ou sans la particule von] »), passant ainsi sous silence la cristallisation de la topolignée autour de ses terres (voir infra, note 15).

${ }^{10}$ La chronologie des types sigillographiques féminins est difficile à préciser en raison de l'hétérogénéité d'un corpus en définitive assez difficile à cerner mais numériquement peu important et où des types différents continuent d'être utilisés indépendamment de leur date de première apparition - ainsi le type de la « femme-pont » utilisé encore en plein $\mathrm{XV}$ e siècle alors que le type héraldique présentant les seuls écu et patronyme du père (en plus du prénom de la femme) semble dominer depuis le tournant du siècle. Une étude de grande ampleur serait nécessaire, constituant un aspect de l'étude de la parenté aristocratique à la fin du Moyen Âge, à laquelle j'entends me consacrer plus intensivement.

${ }^{11}$ Bayerisches Staatsarchiv Würzburg, Würzburger Urkunden, Nr 45/109c (1489).

${ }^{12}$ Il faut toutefois tenir compte du fait que d'autres types continuent d'être utilisés et que le mode de corroboration change au XVIe siècle avec l'avènement de la signature, qui reprend la forme anthroponymique utilisée dans le texte et la juxtapose à un sceau sans légende mais présentant les armes du père et du mari. 


\section{b. Patronymes masculins : le lignage quand même}

Dans ces conditions, on doit s'interroger sur le sens de ce qui semble avoir été chez les hommes une tentative vaine d'introduction à la fin du XIVe siècle d'un système anthroponymique triple proche de celui observé chez les femmes. On observe en effet dans le dernier quart du XIVe siècle des mentions de nobles [95] désignés par leur prénom et leur patronyme, suivi de la précision « dit [patronyme de la mère] » (par exemple Hans von Thüngen, genannt von Eisenbach), sans que la mention du lignage maternel corresponde à une hypergamie masculine, ni à une mère unique héritière, ni à une nécessité de différencier entre plusieurs porteurs du même prénom au sein d'un lignage (pour lesquels on utilise l'indication de l'âge relatif ou de la résidence ${ }^{13}$ ). Ces mentions, contemporaines de l'usage des sceaux du type "femme-pont », semblent bien aller dans le même sens que ce qui apparaît à l'examen des pratiques patronymiques et sigillographiques des femmes, à savoir la dicibilité, désormais, du lignage maternel. Toutefois, cette pratique anthroponymique n'a pas eu de suite et le $\mathrm{XV}$ siècle nous montre des hommes désignés exclusivement par leur prénom et le patronyme de leur père.

On est donc là en présence de l'un de ces intéressants cas de représentations marginalisées, susceptibles de renvoyer à ces rapports sociaux existant idéellement à l'état de satellite des rapports existant, eux, concrètement ${ }^{14}$, et aussi de marquer, par leur marginalité même, jusqu'où le système social dans lequel elles apparaissent comme marginales est prêt à admettre des inflexions et adaptations. Bref, il semble que l'échec historique de cette alternative anthroponymique fasse ressortir ce qui, fondamentalement, ne pouvait (ou ne devait) pas être touché : l'idéologie lignagère (patrilinéaire ${ }^{15}$ ). L'abandon de la mention du lignage maternel exprime ainsi un discours patrilinéaire, le discours lignager lui-même (ce qui ne signifie pas que le système de parenté soit patrilinéaire). La concentration des hommes sur le patronyme de leur père doit [96] donc être considérée comme un choix discursif, non comme la simple mise à jour d'une tendance lignagère à l'œuvre depuis quelques siècles : une alternative anthroponymique a existé et été reconnue, mais elle a été finalement rejetée au profit d'une autre pratique qui réalisait le même objectif mais ne mettait pas en cause l'idéologie patrilinéaire courante dans l'aristocratie.

Cette cristallisation patronymique des hommes doit donc être rangée parmi les phénomènes qui constituent la mutation patronymique complexe dont j'ai parlé. Elle permet en outre de préciser la portée du changement perçu pour les femmes: la mention de la double appartenance des femmes ne doit certainement pas être conçue comme l'expression d'un affaiblissement du discours lignager (qui se veut

\footnotetext{
${ }_{13}$ Chez les Thüngen, par exemple, on trouve ainsi en 1371 des enfants gnant von Seinßheim, nés du mariage de Dietrich von Thüngen et de Gerhaus von Seinsheim (Bayerisches Staatsarchiv Würzburg, Archiv Thüngen zu Weißenbach, Bände, $\left.\mathrm{Nr} 1, \mathrm{f}^{\circ} 19 \mathrm{r}^{\circ}\right)$. Critères complémentaires de distinction d'homonymes : âge relatif (en 1372, on trouve un Eherhard von Rosenberg der Junge genannt von Thüngen: Wilhelm ENGEL (éd.), Würzburger Urkundenregesten vor 140o, Würzburg, 1958, $\mathrm{Nr}$ 163), résidence (on trouve en 1393 un Hans von Thüngen gesessen zum Reußenberg, den man nennt von Eisenbach: Bayerisches Hauptstaatsarchiv München, Würzburger Urkunden, Nr 2596, éd. fautive Monumenta Boica, t. 44, München, 1883, Nr 148).

14 Voir M. GODELIER, L'idéel et le matériel..., p. 223-227.

15 Wolfhard VAHL, Die Siegel des niederen fränkischen Adels im 13. und 14. Jahrhundert, Erlangen-Nürnberg: ms. dactyl. microfiché (Dissertation), 1993, p. 125-126, a observé sur les sceaux des petits nobles de Haute-Franconie (dans la région dite Fränkische Schweiz) un intéressant phénomène concernant les patronymes masculins au XIVe siècle : un passage (temporaire, mais non pour autant dénué de signification) de la forme "von (ou de) [toponyme] » à la forme "[toponyme]er» (par exemple de von Forchheim à Forchheimer), génitivation d'un substantif qui manifesterait - à l'instar du gentilice romain (mais aussi de ce qu'Olivier Guyotjeannin a observé encore dans l'Émilie du XIe siècle) - non plus l'origine ou la résidence mais l'appartenance à un groupe de filiation issu d'un individu nommé, lui, «von [toponyme] ». Bref, le toponyme serait transformé en nomen patrum, comme si la référence à la terre éponyme était éclipsée par la référence aux ancêtres, transfert du spatial au temporel faisant du lignage non une topolignée mais une chronolignée; le retour à la forme von/de $N^{*}$ pourrait être le signe d'un changement accompli plutôt que de son échec - le XIVe siècle apparaissant ainsi comme un moment important de la maturation de l'identité lignagère des topolignées - mais la mesure et l'interprétation correctes du phénomène nécessitent toutefois des études encore plus poussées.
} 
patrilinéaire), puisque parallèlement on assiste à la concentration des hommes sur leur patronyme et que l'on doit sans doute, en bonne logique, considérer l'apposition sur la femme mariée ${ }^{16}$ d'une "marque d'origine " indélébile (le patronyme du père) comme une expression du renforcement du principe d'appartenance lignagère. Et comme tout ceci fait l'objet non pas (ou pas seulement) de pratiques orales (dont nous n'avons plus trace) mais scripturales, et qu'en outre le phénomène est général dans la petite noblesse franconienne, on doit en déduire qu'il s'agit moins d'un problème de conscience parentélaire (savoir avec qui on est allié) que de dramaturgie matrimoniale lignagère (montrer à tous à qui le lignage est allié à travers le mariage de deux êtres) - en soi équivalente à l'élaboration de généalogies (activité qui semble pratiquement, sinon totalement, absente dans la petite noblesse).

Cette dimension dramaturgique est selon toute vraisemblance au principe de la figuration, de plus en plus fréquente au $\mathrm{XV}^{\mathrm{e}}$ siècle, des armoiries des quatre aïeuls sur les plaques tombales: le but ici recherché est certainement moins de prouver la noblesse du mort (dont la désignation anthroponymique correspond à celle en usage pour son sexe dans les textes) ni son «identité lignagère » (connue par le patronyme et opposée à la mention des aïeuls matrilinéaires/maternels) que de montrer son intégration parentélaire et sociale ${ }^{17}$. On devrait alors comprendre l'adoption d'un principe de décompte [97] de la noblesse par quartiers (contrairement à la pratique du décompte par degrés en France, par exemple) comme l'expression d'une priorité donnée au principe de la largeur d'intégration dans le groupe sur le principe d'ancienneté masculine de la noblesse (qui est à l'arrière-plan du décompte par degrés). La triple dénomination de la femme (son double patronyme) irait donc dans le sens de cette composition visible d'un groupe noble, composé non par la juxtaposition de lignages nobles mais par leur intégration matrimoniale, laquelle fait alors l'objet d'une mise en discours propre à faire connaître et reconnaître la constitution du groupe qui commence alors seulement à se désigner et être désigné comme « noblesse » (Adel) ${ }^{18}$.

\section{Nouveaux usages du prénom}

Parallèlement à ces nouveautés dans le domaine patronymique - qui posent le problème du rapport entre l'usage courant et les usages scriptural et sigillographique -, de notables inflexions peuvent être observées dans le champ du prénom nobiliaire.

\section{a. Les prénoms communs de la noblesse}

Les $\mathrm{XIV}^{\mathrm{e}}$ et $\mathrm{XV}^{\mathrm{e}}$ siècles sont marqués par une profonde recomposition du stock des principaux prénoms utilisés. Comme le montre le tableau $1^{19}$, il ne reste dans la

\footnotetext{
${ }^{16}$ Rappelons que les femmes mariées peuvent souvent ne plus se distinguer sigillographiquement des femmes non mariées.

${ }^{17}$ Le cas de la confrérie de sœurs de l'Ancolie (Agelblume-Schwesternschaft) est à cet égard éclairant. Le texte de (re)fondation en 1498 (Würzburger Provinzialarchiv der deutschen Augustiner, U 155), fait apparaître 24 dames nobles, toutes nommées de manière triple $\left(\mathrm{P}^{*} \mathrm{~N}^{*}\right.$, née $\left.\mathrm{N}^{* *}\right)$. Or, les statuts précisent que lorsqu'une candidate à l'entrée n'est pas mariée, elle doit déclarer ses quatre aïeuls (voir Norbert BACKMUND, Die kleineren Orden in Bayern und ihre Klöster bis zur Säkularisation, Abtei Windberg: Poppe-Verlag, 1974, p. 21). Cette déclaration ne peut être considérée comme la réponse à une exigence de naissance noble, puisqu'elle est substitutive du mariage des femmes, lequel n'est pas une garantie de noblesse. En revanche, il est clair que l'indication du réseau de naissance équivaut à l'indication du réseau de mariage. La déclaration des quatre aïeuls apparaît ainsi, pour les femmes non mariées, comme l'équivalent structurel de la double désignation anthroponymique, dont la fonction est finalement de manifester la position dans le groupe noble.

${ }^{18}$ Sur le sens et la portée de cette nouveauté terminologique, voir J. MORSEL, « Die Erfindung... ».

19 Les tableaux qui suivent ne retiennent que les prénoms qui forment la première médiale de la série étudiée à la date indiquée (les prénoms entre parenthèses appartiennent à la seconde médiale mais sont ainsi signalés afin de mieux suivre leur progression). Les huit premières séries du tableau 1 sont données de manière plus complète dans ma contribution «De l'usage politique et social du prénom en Franconie à la fin du Moyen Âge », in : Philippe CONTAMINE et alii (dir.), Commerce, finances et société (XI ${ }^{e}-X V I^{e}$ siècles). Recueil de travaux d'Histoire médiévale offert à M. le Professeur Henri Dubois, Paris : Presses de Paris-Sorbonne, 1993, p. 379-393. Les séries de 1303-1313, 1335-1345,
} 
première médiale en 1525 que deux prénoms sur les sept représentés en 1303-1313. Et sur les dix prénoms qui composent cette première [98] médiale en 1525, un seul mais de taille est apparu au XIV e siècle (Johann), tandis que tous les autres ont acquis leur importance au XVe siècle. Si le premier phénomène (Johann dépassant Heinrich et Konrad) est perceptible également chez les non-nobles (voir tableau 2) ${ }^{20}$ et correspond - quoiqu'avec un certain retard ici - à un phénomène général dans l'Occident chrétien ${ }^{21}$, le second présente des caractères propres à la noblesse.

\section{Tableau 1 : Évolution du groupe des principaux prénoms des nobles du début du XIVe au début du XVI'e siècle}

\begin{tabular}{|c|c|c|c|c|c|c|c|c|c|}
\hline rang & $\begin{array}{c}1303- \\
1313 \\
\end{array}$ & $\begin{array}{l}1335- \\
1345 \\
\end{array}$ & v. 1388 & $\begin{array}{c}1400- \\
1411\end{array}$ & 1449 & $\begin{array}{l}1455^{-} \\
1466 \\
\end{array}$ & 1485 & $\begin{array}{c}1495- \\
1511 \\
\end{array}$ & 1525 \\
\hline 1 & Heinrich & Heinrich & Johann & Johann & Johann & Johann & Johann & Johann & Johann \\
\hline 2 & Konrad & Konrad & Heinrich & Heinrich & Heinrich & Georg & Georg & Georg & Georg \\
\hline 3 & Friedrich & $\begin{array}{c}\text { Friedrich } \\
\text { Johann }\end{array}$ & Konrad & Konrad & Konrad & Heinrich & Heinrich & Phipp & Wolfram \\
\hline 4 & Hermann & & Friedrich & Friedrich & Georg & Konrad & Konrad & Wilhelm & Wilhelm \\
\hline 5 & Gottfried & Hermann & & Dietrich & Friedrich & Peter & $\begin{array}{l}\text { Wilhelm } \\
\text { Philipp }\end{array}$ & Heinrich & $\begin{array}{l}\text { Christoph } \\
\text { Sigmund } \\
\end{array}$ \\
\hline 6 & $\begin{array}{c}\text { Berthold } \\
\text { Otto }\end{array}$ & Berthold & & Peter & Wilhelm & Friedrich & & Christoph & \\
\hline 7 & & & & Eberhard & Ulrich & Wilhelm & $\begin{array}{l}\text { Sigmund } \\
\text { Christoph }\end{array}$ & Konrad & Philipp \\
\hline 8 & & & & & & Eberhard & & Wolfram & Bernhard \\
\hline 9 & & & & & & & $\begin{array}{c}\text { Friedrich } \\
\text { Michael } \\
\end{array}$ & Sigmund & $\begin{array}{l}\text { Heinrich } \\
\text { Friedrich } \\
\end{array}$ \\
\hline 10 & (Johann) & & & (Georg) & & & & Kaspar & \\
\hline 11 & & & & & (Philipp) & (Martin) & Dietrich & $\begin{array}{c}\text { Martin } \\
\text { Jakob } \\
\text { Friedrich } \\
\end{array}$ & \\
\hline$n$ & 788 & 522 & 381 & 742 & 868 & 534 & 278 & 832 & $\mathbf{2 5 7}$ \\
\hline
\end{tabular}

1400-1411, 1455-1466 et 1495-1511 ont été obtenues à l'aide du traitement informatique des registres de fiefs wurtzbourgeois (voir supra, note 4). Les séries de ca. 1388, 1449, 1485 et 1525 correspondent, elles, à des listes établies à l'occasion d'événements précis : déclaration de guerre (non datée) de nobles franconiens contre la ville de Schweinfurt (mal datée et interprétée par l'éditeur du texte Friedrich STEIN (éd.), Monumenta Suinfurtensia historica [...], Schweinfurt, 1875, Urkunde $\mathrm{Nr}$ 187, mais dont une datation et interprétation meilleures sont permises par son rapprochement avec la charte cataloguée par J. KoCH et alii (éd.), Regesten àer Pfalzgrafen am Rhein 1214-1508, t. 1, Innsbruck, 1894, Nr 6725); déclaration de guerre des nobles alliés au margrave de Brandebourg-Ansbach contre Nuremberg (voir supra, note 4); participants au tournoi d'Ansbach (voir supra, note 4); relevé des dommages provoqués par les paysans soulevés lors de la Guerre des Paysans de 1525 (voir supra, note 4). Ces séries sont donc loin d'être homologiques, de même qu'elles sont d'importance numérique très variable $(n=$ population statistique composant la série), ce qui peut avoir des effets non négligeables étant donné la logique statistique de la recomposition du stock des prénoms.

${ }^{20}$ Les quatre premières séries du tableau 2 sont données de manière plus complète dans : J. MORSEL, « De l'usage politique et social... », tableaux 2, 4, 7 et 11. Les trois premières ont été obtenues à partir des registres de fiefs wurtzbourgeois (voir note précédente). Celle de 1467-1477 a été construite par exploitation informatique du premier censier de l'évêché de Wurtzbourg (voir supra, note 4). Celle de 1500-1550 résulte du comptage des dépendants à Veitshöchheim de l'abbaye bénédictine St. Stefan de Wurtzbourg sur la base de l'index du censier de l'abbaye établi dans le première moitié du XVI ${ }^{e}$ siècle (Bayerisches Staatsarchiv Würzburg, Standbücher, Nr 626). Johann connaît chez les non-nobles un développement semblable à celui observé chez les nobles, mais peut-être plus précocement (comparer les séries de 1303-1313) et en tout cas plus fortement chez les non-nobles, où sa part de $n$ s'accroît durant le XVe siècle $(24,26,8$ et $30 \%$ pour les trois dernières série), alors qu'elle culmine chez les nobles à $21 \%$ vers 1388 et décline jusque vers $12 \%$ au début du XVI ${ }^{\mathrm{e}}$ siècle (11 \% en 1495-1511, $13 \%$ en 1525).

${ }^{21}$ Sur le développement des prénoms johanniques en Occident, voir Jean-Louis BigET, « L'évolution des noms de baptême en Languedoc au Moyen Âge (IXe-XIVe s.) », Cahiers de Fanjeaux, 17 (Liturgie et Musique), 1982 , p. 316-331. 


\section{Tableau 2 : Évolution du groupe des principaux prénoms des non-nobles du début du XIVe au début du XVI' ${ }^{\mathrm{e}}$ siècle}

\begin{tabular}{|c|c|c|c|c|c|}
\hline rang & $\begin{array}{c}1303- \\
1313 \\
\end{array}$ & $\begin{array}{c}1335- \\
1345 \\
\end{array}$ & $\begin{array}{c}1400- \\
1411 \\
\end{array}$ & $\begin{array}{c}14 S 7- \\
1476\end{array}$ & $\begin{array}{c}1500- \\
1550 \\
\end{array}$ \\
\hline 1 & Heinrich & Heinrich & Johann & Johann & Johann \\
\hline 2 & Konrad & Konrad & Konrad & Konrad & Nikolaus \\
\hline 3 & Hermann & Johann & Heinrich & Heinrich & Georg \\
\hline 4 & $\underline{\text { Friedrich }}$ & $\underline{\text { Friedrich }}$ & & $\underline{\text { Nikolaus }}$ & $\underline{\text { Andreas }}$ \\
\hline 5 & & & & & (Konrad) \\
\hline \multicolumn{6}{|l|}{6} \\
\hline 7 & (Johann) & & & (Andreas) & \\
\hline \multicolumn{6}{|l|}{$\boldsymbol{8}$} \\
\hline 9 & & & (Nikolaus) & (Georg) & \\
\hline 10 & & & (Andreas) & & \\
\hline$n$ & 410 & 416 & 752 & 3645 & 246 \\
\hline
\end{tabular}

En effet, on remarque au XVe siècle le triomphe net du prénom Georg, qui déloge les prénoms « impériaux » Heinrich et Konrad et réduit peu à peu son écart avec Johann (de 19:6 en 1449 à 13:9 en 1525). Or, la connotation chevaleresque du prénom ne fait aucun doute, comme en témoigne les mentions fréquentes de "Georges le saint chevalier » dans les formules de datation qui reposent sur le jour de sa fête ${ }^{22}$, les représentations iconographiques de saint Georges et surtout les très nombreux choix de ce saint comme patron de sociétés de chevalerie ${ }^{23}$. De plus, Georg est suivi d'autres prénoms de saints chevaliers, comme Wilhelm, Martin, Jakob, Michael, Moritz, Sebastian, Florian, Cyriak, Adrian, Pankraz, etc. et, chez les femmes (pour autant que les [100] maigres chiffres permettent de le savoir), Barbara, Margaretha, etc. ${ }^{24}$ Ces saints chevaliers fournissent chez les hommes un tiers des prénominations (pour un sixième des prénoms) au début du XVIe siècle. Enfin, ces prénoms n'atteignent pas du tout la même importance chez les non-nobles, où Konrad reste à son second poste jusqu'à la fin du $X^{\mathrm{e}}$ siècle (contribuant ainsi sans doute à connoter le prénom de

\footnotetext{
${ }^{22}$ L'insistance particulière sur la dimension chevaleresque de saint Georges dans les formules de datation est en outre confirmée par le fait qu'il n’y est jamais désigné comme « saint Intercesseur » (voir H. GROTEFEND, Taschenbuch der Zeitrechnung, $10^{\mathrm{e}}$ éd. Hannover, 1960, p. 60), alors qu'il fait partie des Quatorze Intercesseurs (Vierzehn Nothelfer) dont la fortune est grande dans l'Empire à la fin du Moyen Âge; toutefois, d'après Gerd ZIMMERMANN, «Patrozinienwahl und Frömmigkeitswandel im Mittelalter, dargestellt an Beispielen aus dem alten Bistum Würzburg (Teil II) », Würzburger Diözesan-Geschichtsblätter, 21, 1959, p. 93, le culte des Quatorze Intercesseurs ne s'est vraiment répandu en Franconie qu'à partir du XVIe siècle - à quoi correspond peut-être une partie du succès de Georg chez les dépendants de Veitshöchheim en 1500-1550 - sans toutefois que la dimension chevaleresque disparaisse sans doute jamais, comme semble l'indiquer un passage du traité de Jean Molanus, De Picturis et Imaginibus (Louvain, 1570), p. 278 (sur lequel mon attention a été attirée par Olivier Christin). Le cas de Georges manifeste ainsi clairement les problèmes d'identification de l'éponyme (ou de l'aspect de l'éponyme), en raison de la polysémie des saints et des variations régionales et sociales des cultes qui leur sont rendus.

${ }_{23}$ Holger KRUSE et alii, Ritterorden und Adelsgesellschaften im spätmittelalterlichen Deutschland, Frankfurt/Bem/New York/Paris : Peter Lang (Kieler Werkstücke, D, 1), 1991, p. 26 : saint Georges est de très loin le saint le plus fréquemment choisi comme patron dans l'Empire et les sociétés qui le choisissent sont principalement localisées dans le Sud-Ouest de l'Empire (c'est-à-dire dans ces régions dont l'évolution socio-politique m'intéresse ici) et en Autriche.

24 Sur les saints patrons chevaleresques, voir Dietrich H. Kerler, Die Patronate der Heiligen, (Ulm, 1905) réimp. Hildesheim : Georg Olms, 1968, p. 184-185, 220-221, 293-297, 338-339 et, spécifiquement pour la Franconie, G. ZimmermanN, "Patrozinienwahl... », p. 38-6o. La diffusion des prénoms chevaleresques est perceptible sur les planches généalogiques des Seinsheim et des Thüngen en annexe (voir aussi le commentaire, § III.2).
} 
rusticité25) avant d'être remplacé par un Nikolaus dont on suit la progression durant tout le $\mathrm{XV}^{\mathrm{e}}$ siècle, alors qu'il reste presque complètement absent des prénoms portés par les nobles.

On a donc bien affaire à la mise en place de régimes de prénomination socialement différenciés, celui des nobles étant fortement connoté à la chevalerie. Or, la référence à la chevalerie dans la noblesse franconienne de la fin du Moyen Âge ne peut être considérée comme l'expression d'une fuite de celle-ci dans un idéal sans villes et sans princes. Il apparaît en effet qu'un modèle (et non pas un idéal ${ }^{26}$ ) chevaleresque est construit dans la noblesse franconienne de la fin du Moyen Âge, à coup d'ordonnances normatives (pour participer aux grands tournois, pour entrer dans les sociétés de tournoi, etc.) ${ }^{27}$, mais aussi de représentations funéraires (les nobles se font tous représenter en armure) ou historico-littéraires (Ludwig von Eyb, Götz von Berlichingen, hérauts d'armes, etc.), et que ce modèle s'avère être un moyen idéologique d'absorption du prince par et dans la noblesse ${ }^{28}$. La diffusion des prénoms de [101] saints chevaliers pourrait ainsi apparaitre comme le versant anthroponymique de la genèse d'une idéologie chevaleresque, dont il permettrait de dater les prodromes dès les alentours de 1400 - précisément le moment auquel la Ritterschaft ( ensemble des chevaliers ») devient la désignation discursive de la petite noblesse en action ${ }^{29}$ : anthroponymie et terminologie se renouvelleraient ainsi conjointement l'anthroponymie n'étant finalement qu'une variante de la terminologie...

\section{b. Prénoms nobiliaires : le lignage quand même}

Toutefois, dès que l'on examine la diffusion des prénoms chevaleresques au sein des topolignées prises individuellement, on se rend compte que, d'une part, ils n'occupent qu'une place assez secondaire par rapport à leur position statistique globale (Georg, par exemple, paraît n'être jamais le deuxième prénom le plus fréquent dans chaque génération de la seconde moitié du XVe et du début du XVI ${ }^{\mathrm{e}}$ siècle, ni d'ailleurs Johann le prénom le plus fréquent ${ }^{30}$ ), et surtout qu'ils ne semblent pas remettre en cause une forme patrimoniale (topolinéaire, voire plus globalement linéaire) de circulation des prénoms. Beaucoup de topolignées connaissent en effet l'existence de prénoms qui circulent en ligne masculine (tendanciellement de grand-père en petit-fils, mais sans règle absolue ni d'ailleurs, semble-t-il, différence entre aînés et cadets - pour autant que les informations conservées fassent connaître l'ordre des naissances et aussi les décès en bas âge). Certains de ces prénoms sont étroitement liés à des seigneuries précises, mais d'autres transcendent les diverses topolignées et sont d'ailleurs presque

\footnotetext{
${ }^{25}$ Les révoltes populaires qui se multiplient dans le Sud et le Sud-Ouest de l'Empire depuis le milieu du XVe siècle sont fréquemment désignées, aux environs de 1500, par l'expression armer Konrad, parfois associée à l'expression Bundschuh (chaussure à lanières servant de signe de ralliement aux révoltes depuis le milieu du XVe siècle) : voir par exemple Adolf WREDE (éd.), Deutsche Reichstagsakten unter Kaiser Karl V., t. 3, Gotha, 1901 (DRTA, jüngere Reihe, III), Nr 113, § I-1, p. 697 (wo sich ein puntschuch oder armer Cuntz entporen solt...); tout se passe donc comme si le prénom Konrad connotait les couches populaires désormais tout autant que la chaussure lacée, sur le mode de ce qui s'était passé en France avec le prénom Jacques au moment de la Jacquerie. Johann identifierait alors religieusement (comme chrétien) le stock global (nobiliaire ou non) des prénoms, Konrad et Georg le distribuant socialement, d'où la disparition de Konrad chez les nobles et, peut-être, l'imitation de Georg par les non-nobles (voir supra, note 22).

${ }^{26}$ Voir Carlos BARros, "Cómo vive el modelo caballeresco la hidalguia gallega bajo medieval : Los Pazos de Probén », in: Galicia en la Edad Media (Actas del Coloquio celebrado del 13 al 17 de julio en Santiago de Compostela, A Coruña, Pontevedra, Vigo y Betanzos), Madrid : Sociedad Española de Estudios Medievales, 1990, p. 231-232.

${ }_{27}$ Voir J. MorSEL, "Le tournoi, mode d'éducation politique en Allemagne à la fin du Moyen Âge », in : Éducation, apprentissages, initiation au Moyen Âge (Actes du premier colloque international de Montpellier, nov. 1991), Montpellier : Presses Universitaires, 1993 (Les Cahiers du C.R.I.S.I.M.A., 1), p. 309-331.

${ }^{28}$ Voir la circulaire émise en 1478 pour relancer la pratique du tournoi, éditée par J. MoRSEL, "Le tournoi... », annexe 1. Sur les relations entre prince et chevalerie, voir par exemple Werner PARAVICINI, «Rois et princes chevaliers (Allemagne, XIIe-XVI e siècles) », in : S.H.M.E.S. (dir.). Les princes et le pouvoir au Moyen Âge (XXIII e congrès de la S.HM.E.S., Brest, 1992), Paris : Publications de la Sorbonne, 1993, p. 9-34: en tant que chevalier, le prince n'est plus qu'un primus inter pares, ce qui le conduit souvent à se dégager de son engagement chevaleresque.

29 Sur le sens politique et discursif de Ritterschaft, voir J. MORSEL, « Die Erfindung... ».

${ }^{30}$ Voir infra (planches Seinsheim et Thüngen en annexe et commentaire, § III. 2).
} 
exclusifs aux lignées, qu'ils tendent finalement à identifier presque aussi sûrement que les patronymes: c'est ainsi le cas d'Erkinger chez les Seinsheim, Stamm chez les Schlitz, Bosse chez les Buchenau, Frowin chez les Hutten, Reu $\beta$ chez les Thüngen, ailleurs (dans l'évêché de Spire) Raban chez les Helmstatt, etc. La circulation topolinéaire conduit également - et logiquement - à l'introduction dans les lignées de nouveaux prénoms liés à des héritages importants venus par les femmes épousées, ce qui d'une part souligne symboliquement la continuité patrimoniale d'un lignage à l'autre, exprime d'autre part la logique terrienne (depuis les $\mathrm{X}^{\mathrm{e}}$-XII ${ }^{\mathrm{e}}$ siècles) de l'anthroponymie aristocratique, enfin semble montrer que certains prénoms apparaissent plus proprement "identifiants » (plus liés à la terre considérée comme patrimoniale) que d'autres, donc que [102] certains prénoms peuvent avoir une dimension identitaire nette ${ }^{11}$ (les autres pouvant renvoyer par exemple à des fidélités religieuses, féodales ou aristocratiques).

Le changement perçu dans les prénoms relève donc essentiellement d'une logique statistique : les prénoms spécifiques aux lignées et/ou topolignées sont éliminés de la première médiale au fur et à mesure que l'on agrège les lignées, tandis qu'y entrent seuls les prénoms les moins spécifiques (quoique moins nombreux par génération). Les prénoms chevaleresques doivent donc être considérés non comme les plus appréciés par les topolignées aristocratiques, mais comme les plus communément nobles. La production anthroponymique de l'idéologie nobiliaire n'est pas une production directe mise en œuvre (consciemment ou non) par les topolignées, mais repose sur l'agrégation statistique des actions isolées de nomination chevaleresque, assurant ainsi à l'ensemble $\mathrm{du}$ groupe aristocratique une dimension chevaleresque/nobiliaire qu'elle refuse (anthroponymiquement) à tel ou tel de ses éléments (les groupes de filiation) pris séparément. Cette logique statistique révèle à la fois, en négatif, la prégnance du mode de reproduction topolinéaire et, en positif, la diffusion, dans les interstices de celui-ci, d'une idéologie chevaleresque commune à toutes les (topo)lignées nobles. Ceci permet à la fois la reproduction directe d'une conscience lignagère (par le biais de la collation des prénoms traditionnels de la topolignée) et la production/reproduction indirecte d'une idéologie chevaleresque spécifiquement nobiliaire, dont l'effet d'homogénéisation socio-politique est peut-être d'autant plus fort qu'il est justement moins apparent, permettant à la fois une prise de conscience nobiliaire et évitant en même temps la copie pure et simple par les non-nobles. Cette impression de reproduction "intégrée » des structures de l'aristocratie ne doit toutefois pas faire oublier que la logique statistique résulte de l'existence d'une contradiction possible entre le mode de reproduction parental (topolinéaire) et le mode de reproduction groupal (nobiliaire).

\section{Lignage et noblesse : articulation anthroponymique?}

On observe donc à travers l'anthroponymie deux phénomènes qui pourraient sembler contradictoires : d'une part le renforcement de la spécificité lignagère (renforcement patronymique, résistance des prénoms topolinéaires) et de l'autre le développement de pratiques synthétiques nobles (doubles patronymes féminins, prénoms chevaleresques communs, figuration des armes des quatre aïeuls sur la tombe).

[103]a. Rapports de parenté et sociogenèse de la noblesse

Le premier de ces phénomènes correspond à un discours lignager qui vient assurer la transmission des biens patrimoniaux en soumettant les intérêts des individus à des

\footnotetext{
${ }^{31}$ Voir infra (annexe, § I. 3 et II. 1) le cas du prénom Ulrich, entré chez les von Hutten à la suite de l'héritage de biens de la topolignée éteinte en ligne masculine de Steckelberg.
} 
intérêts parentaux conçus comme naturels et armés par une morale de l'honneur ${ }^{32}$. Le second correspond à un discours nobiliaire qui procède de l'exigence d'une organisation politique et idéologique de l'aristocratie face aux autres forces de la région (princes, villes, ruraux), afin de maintenir ou renforcer son pouvoir sur les hommes et la terre, et qui s'exprime sous la forme de ligues, assemblées, co-seigneuries châtelaines (Ganerbenburgen), tournois, etc. Or, ces deux discours devaient être articulés de manière à permettre l'association de lignages que leur identité et leurs intérêts patrimoniaux poussaient à s'opposer33 tout en évitant que ces intérêts et identité soit sacrifiés à cette exigence de l'association.

Cette articulation n'a été rendue possible qu'en dissociant les niveaux patrimonial et matrimonial de structuration de l'aristocratie, en renforçant l'homogénéité et la prégnance du premier et en soulignant la dimension intégratrice du second. Cette dissociation apparaît très clairement lorsque l'on [104] examine les clauses des contrats de mariage. Les données concernant le XIVe siècle sont plutôt rares pour les petits nobles (contrairement à la catégorie des comtes et sires34), mais on peut observer à la fin du XIVe siècle des douaires composés de dîmes, biens-fonds et rentes.

$\mathrm{Au} \mathrm{XV}^{\mathrm{e}}$ siècle, en revanche, les douaires (le matrimoine) apparaissent clairement en lumière : ils comprennent la dot $($ Zugeld $=$ dos) versée par le père, la contre-dot (Gegengeld $=$ dos ex marito) versée par le mari, constituée en rentes comme la dot et d'un montant rigoureusement équivalent, le don du matin (Morgengabe $=$ donatio propter nuptias) fait par le mari à sa discrétion, le trousseau (Heimsteuer ou Fertigung) donné par le père à sa discrétion et un tiers (parfois un demi) des biens meubles - à l'exception expresse de biens que l'on pourrait qualifier de " stratégiques » (armes et armures, créances, argent) : le meuble est donc essentiellement composé de biens domestiques. À l'exception de ceux composant le don du matin et le trousseau, dont la femme a libre disposition, tous les biens reviennent, à la mort de la douairière, à ses fils - les filles mariées ou entrées au couvent devant renoncer à leur héritage paternel, maternel et fraternel en contrepartie de leur dotation.

Le patrimoine est ainsi composé des terres, autres immeubles et biens

${ }^{32}$ Sur la fonction structurante et stabilisatrice du discours familial et de la morale de l'honneur, voir Pierre BouRDIEU, Le sens pratique, Paris : Minuit, 1980, p. 315-325. Sur la morale de l'honneur à la fin du Moyen Âge, voir Claude GAUVARD, «De grâce especial ». Crime, État et société en France à la fin du Moyen Âge, Paris : Publications de la Sorbonne, 1991, notamment t. 2, p. 705-788 ; Kristen B. NeUSCHEL, Word of Honor. Interpreting Noble Culture in Sixteenth-Century France, Ithaca/London : Comell University Press, 1989; Hillay ZMORA, "Ehre und Fehde im fränkischen Adel 1450-1550 », in : Klaus ScHREINER (dir.), Verletzte Ehre, Köln/Weimar/Wien : Böhlau (Norm und Struktur. Studien zum sozialen Wandel im Mittelalter und früher Neuzeit, 4), sous presse.

33 Il n'est pas possible d'examiner ici en détail la logique interne des faides qui opposent souvent les lignées nobles : rappelons seulement qu'elles sont indispensables au fonctionnement et à la reproduction des rapports seigneuriaux, notamment en réactualisant périodiquement la relation de réciprocité déséquilibrée existant entre les deux parties et en construisant dans l'esprit des dépendants une frontière les départageant des dépendants de seigneurs voisins, présentés comme une menace pour la reproduction de la communauté. Mais la guerre entre seigneurs était également essentielle à la cohésion des topolignées (les faides ne sont donc pas seulement l'expression de la cohésion lignagère, à quoi on les réduit le plus souvent, mais elles en sont le moyen, travesti sous forme d'un code de l'honneur dont le respect semble s'imposer de manière naturelle à chaque membre du lignage), ce qui explique qu'elle se soit déroulée dans un cadre prioritairement topolinéaire, ce que garantissait l'imbrication des biens des diverses branches et des divers membres (tant que les topolignées ne sont pas parvenues à discipliner complètement leurs membres et à les soumettre - de façon d'autant plus invisible qu'elle semblera aller de soi - à la logique lignagère et patrimoniale, l'imbrication des biens de chacun reste nécessaire afin d'éviter le repliement de chacun sur ses biens au détriment de la solidarité lignagère ; ceci interdisait donc l'adoption de règles d'unigéniture comme le "droit d'aînesse »). Or, cette imposition d'une discipline lignagère/patrimoniale interne n'est encore qu'incomplètement réalisée à la fin du Moyen Âge, comme le montrent les cas de nobles (pas forcément en position dominée dans leur lignée) qui développent des stratégies " contre-lignagères ». Rien ne justifiait donc, du côté seigneurial comme du côté parental, que les faides se raréfient, en l'absence de princes ayant les moyens (ou la volonté ?) de prendre le « monopole la violence légitime ». La cohésion du groupe noble était donc menacée par la spécificité des intérêts topolinéaires (à la fois seigneuriaux et identitaires), que soutenaient, à dessein ou non, les princes.

34 Voir Karl-Heinz SPIEß, Familie und Verwandtschaft im deutschen Hochadel des Spätmittelalters, 13. bis Anfang des 16. Jahrhunderts, Stuttgart : Franz Steiner (VSWG, Beiheft 111), 1993, qui traite pour l'essentiel des comtes et sires de l'Allemagne moyenne (Hesse notamment). Étant donné le caractère non aléatoire de la scripturalité (tout comme de la conservation des documents écrits), la brusque apparition des contrats de mariages aux alentours de 1400 dans la petite noblesse est un phénomène selon toute vraisemblance significatif du point de vue de la production d'un discours sur la noblesse. 
«stratégiques », transmissibles exclusivement par voie masculine. Le matrimoine est, lui, composé de rentes et biens domestiques. D’après la logique des contrats de mariage, la dotation des filles et la contre-dotation des épouses des fils ne peut se faire structurellement qu'à partir des biens matrimoniaux35. On assisterait donc à la mise à jour d'un double système de circulation de biens ${ }^{36}$. [105] Toutefois, la différenciation des deux systèmes ne repose pas seulement sur l'existence de deux classes de biens (matrimoniaux $=$ rentiers/domestiques $v s$. patrimoniaux $=$ fonciers/stratégiques), mais également de deux formes de circulation de biens. La circulation des biens patrimoniaux est tendanciellement «distinctive »: but du discours lignager (la patrimonialisation de la terre), elle aboutit à une identification récurrente de certaines terres avec certains noms et armes. La circulation des biens matrimoniaux, elle, est tendanciellement «intégrative ». Dot et contre-dot ne peuvent pas être interprétées à l'aide des catégories du don/contre-don: l'équivalence et la simultanéité strictes prévues par les contrats de mariage s'opposent en effet à la marge de réponse (délai et valeur du contre-don) laissée au donataire, dans laquelle réside la valeur symbolique du contre-don adéquat (c'est-à-dire socialement équivalent au don)37 - c'est en revanche au trousseau et au don matitudinal, explicitement laissés à la discrétion des partenaires et fondés sur une morale de l'honneur, que revient la tâche délicate de commensurer symboliquement les deux parentèles. Dot et contre-dot ne définissent donc pas la position des topolignées les unes par rapport aux autres, mais la position de la femme par rapport aux unes et aux autres. La dot est normalement conçue comme la compensation forfaitaire de la déshérence de la femme mariée : la femme dotée n'a en échange plus rien à attendre de sa topolignée de naissance. Le fait que son mari ajoute à cela l'exact équivalent doit donc être conçu comme une manière de signifier qu'elle n'a plus non plus quoi que ce soit à attendre matériellement de lui ${ }^{3}$. La femme est donc placée matériellement entre les deux topolignées : elle reçoit autant de son père que de son mari et est donc [106] théoriquement autant redevable à l'un qu'à l'autre - c'est-àdire qu'elle est neutralisée. Et comme les biens matrimoniaux sont neutralisés par leur

35 Ce système rappelle étrangement celui que Tacite présentait pour les Germains du premier siècle : dotem non uxor marito, sed uxori maritus offert. [...] in haec munera uxor accipitur, atque in vicem ipsa armorum aliquid viro affert: hoc maximum vinculum, haec arcana sacra, hos coniugales deos arbitrantur. [...] accipere se quae liberis inviolata ac digna reddat, quae nurus accipiant rursusque ad nepotes referantur. (P. Cornelius TACITUS, Germania, $\S 18$ [éd. Allan A. LUND, Heidelberg : Carl Winter, 1988, p. 84]) : les biens matrimoniaux venus du mari circulent donc de génération en génération et de sexe en sexe (mari $\rightarrow$ femme $\rightarrow$ enfants (fils) $\rightarrow$ brus). La thèse simpliste de la continuité germanique étant inadmissible sans examen (voir l'évolution décrite par Régine LE JAN-HENNEBICQUE, "Aux origines du douaire médiéval (VIe-Xe siècles) », in : Michel PARISSE (dir.), Veuves et veuvage dans le haut Moyen Âge, Paris : Picard, 1993, p. 107-121, qui observe notamment une disparition complète du Morgengabe dans le mariage franc du $\mathrm{X}^{\mathrm{e}}$ siècle), tout jugement hâtif doit être évité quant aux raisons du maintien ou de la réinstitution d'une telle structure de circulation des biens matrimoniaux.

${ }_{36}$ J'insiste sur le fait que ce qu'il y a de neuf dans ces pratiques dotales est qu'elles font désormais l'objet de formalisations textuelles dans la petite noblesse. Dire qu'elles n'existaient pas antérieurement est risqué en l'absence de textes, mais l'important est précisément qu'elles n'ont pas fait l'objet de mises par écrit avant les alentours de 1400 (ce qui m’a été confirmé pour la Fränkische Schweiz par Wolfhard Vahl, que je remercie cordialement), le recours à l'écrit ne pouvant être considéré comme une simple innovation technique : en tant que formalisation d'un rapport social, la rédaction d'un contrats de mariage doit être considérée en tant que telle comme un discours sur le rapport social nommé " mariage » (freundschaft dans les textes en question, où ehe apparaît comme la sanction ecclésiastique de la freundschaft), comme une mise en scène relevant d'une logique sociale nouvelle par rapport à celle qui animait des alliances matrimoniales discrètes. C'est ici tout le problème de la scripturalité qui est posé.

37 Voir Pierre Bourdieu, Esquisse d'une théorie de la pratique, précédée de trois études d'ethnologie kabyle, Genève : Droz, 1972, p. 222-228, et du même. Le sens pratique, p. 167-189 (chap. « L'action du temps »).

${ }^{38} \mathrm{La}$ contre-dot ne peut en effet être réduite à une nécessaire contribution des lignages à l'entretien des filles des autres lignages du fait de la faiblesse financière de ceux-ci puisque, arithmétiquement, une dot (versée à une fille du lignage) et une contre-dot (versée à une femme épousée) sont aussi lourdes qu'une double dot (celle qu'il faudrait verser à une fille du lignage dans un système sans contre-dot). J'insiste sur le fait qu'il ne faudrait pas interpréter tout ceci comme la mise en place d'un nouveau droit matrimonial : ces mesures expriment en fait un effort de discipline interne des lignées, plus ou moins poussé selon les marges de négociation lors de la conclusion des mariages ; on a ainsi des contrats qui prévoient explicitement que la fille et son époux pourront renoncer à la dot lors du partage successoral afin de pouvoir prendre part à celui-ci : même si ces contrats aboutissent finalement au même résultat qu'auparavant (le partage successoral entre tous les enfants, y compris les filles mariées, et donc à la sortie des biens de la lignée), il n'en reste pas moins qu'ils ont d'abord procédé à un discours articulant sans les mélanger les niveaux patrimonial et matrimonial. 
composition équilatérale, ils peuvent être transférés aux fils sans que ceux-ci en soient redevables à la parentèle maternelle. La circulation des biens matrimoniaux, qui passent d'une topolignée à l'autre et d'un sexe à l'autre, est donc une circulation intégrative, qui se surimpose à la circulation patrimoniale sans la perturber.

On observe donc la formalisation d'un double système de transmission successorale des biens, caractérisé structurellement non seulement par son dimorphisme sexuel (classes de biens et types de circulation spécifiques), mais surtout par la construction de positions masculines et féminines différentes par rapport aux topolignées. Étant donné la nécessité de l'association des nobles sans que cela nuise aux intérêts patrimoniaux, le mouvement associatif ne pouvait aller de pair sans un renforcement parallèle de la forme de représentation de la parenté qui semble homothétique au patrimoine : le lignage. D'où un renforcement du discours intégrateur lignager 39 mais aussi, indissociablement, pour éviter le refermement sur soi des unités lignagères, le choix discursif d'un modèle matrimonial de structuration sociologique (aboutissant ainsi à une objectivation d'un régime d'échange généralisé) et passant donc par une neutralisation symbolique de la femme entre son lignage de naissance et son lignage de mariage. Il semble ainsi que, étant donné les contraintes que le système social (importance des topolignées) faisait peser sur les choix de formes d'organisation socio-politiques (qui doivent respecter les topolignées), la nécessité de l'articulation des discours lignager et nobiliaire a [107] conduit à une remarquable reformulation discursive des rapports de parenté, dissociant clairement ceux reposant sur la filiation et ceux sur l'alliance, dissociation matérialisée (c'est-à-dire à la fois exprimée et construite) par une réorganisation de la transmission successorale des biens, corollaire d'une modification des usages patronymiques et sigillographiques à l'endroit des femmes.

\section{b. Sociogenèse de la noblesse, rapports de parenté et anthroponymie}

La nouvelle patronymie des femmes formalise la fonction de «pont » qu'elles occupent non seulement du point de vue matrimonial (alliance entre deux groupes de filiation) mais aussi de la transmission des biens matrimoniaux. La patronymie des hommes exprime de son côté, tout comme la sigillographie dominante des femmes, le renforcement du discours lignager. Inversement, la diffusion très générale des prénoms chevaleresques dans les interstices de la prénomination lignagère semble correspondre à l'inclusion des lignages dans une matrice nobiliaire. Mais ces changements anthroponymiques, remarquablement concomitants, doivent-ils n'être perçus que comme des expressions du changement sociogénétique?

La formation du groupe social intitulé désormais «noblesse » supposait deux

\footnotetext{
${ }^{39}$ Le renforcement du discours lignager est perceptible du point de vue lexical (fréquence et redondance des expressions désignant le lignage: voir supra, note 9); féodal (succession agnatique des Mannlehen nobles : voir Hans-Peter BaUM, Der Lehenhof des Hochstifts Würzburg im Spätmittelalter (1303-1519). Eine rechts- und sozialgeschichtliche Studie, Würzburg : ms. dactyl. (Habilitationsschrift), 1990, t. 1, p. 94-96, et ma thèse citée supra, note 3, p. 263-270) ; patrimonial (multiplication des indivisions successorales et archivistiques : voir Rolf SPRANDEL, " Die Ritterschaft und das Hochstift Würzburg im Spätmittelater », Jahrbuch für fränkische Landesforschung, 36, 1976, p. 123-124); sigillographique (femmes n'arborant que le nom et les armes de leur père, expression angeborenes Insiegel) ; parental (bouclages consanguins au-delà du quatrième degré canon : voir ma thèse citée supra, note 3, p. 505-508, ainsi que le tableau généalogique des Hutten présenté en annexe); terminologico-parental (accentuation de la distinction entre consanguins et affins - avec une nette préférence, au niveau du discours, pour les consanguins : l'étude de la terminologie de la parenté - dans des textes qui, rappelons-le, sont officiels et/ou émis par des hommes pour des hommes, donc nous livrent la lecture masculine/dominante d'une relation le plus souvent à plusieurs faces - fait nettement apparaître qu'au-delà du deuxième degré canon (concernant les parents, enfants, germains, beaux-parents, gendres/brus, beaux-frères et belles-sœurs), on préfère suivre le canal de la consanguinité - lorsque c'est possible sans trop de difficultés - pour se positionner par rapport à un parent; on se désigne alors de manière générique comme vetter, "cousin », même lorsque celui-ci est plus proche, en termes de degrés canons de parenté, par mariage et pourrait alors être désigné de manière générique comme schwager, «beau-frère ». Sur cette terminologie de la parenté, voir ma thèse citée supra, note 3, p. 587-600 ; dans un même ordre d'idées, voir P. BouRdiEU, Le sens pratique, p. 289-290).
} 
formes indissociables de travail social : un travail de composition (regroupement des nobles) et un travail d'imposition (délimitation légitime du groupe vers l'extérieur). Or, ces deux types de travail ont exigé à chaque fois des procédures de nomination, permettant d'identifier et de spécifier les éléments faisant l'objet du regroupement. La structuration discursive du groupe noble reposant sur le lien matrimonial entre les lignages, l'identité de ceux-ci a fait l'objet d'une formalisation accrue, afin de rendre visible (scripturalement du moins) leur chaînage matrimonial (à la manière des sœurs de l'Ancolie, par exemple). Le patronyme a ainsi été l'objet de manipulations qui, par ajustements successifs, l'ont rendu adéquat à son usage sociologique. Par concentration chez les hommes, par évocation chez les femmes et sans oublier le maintien de traditions topolinéaires en matière de prénomination, l'image d'un lignage patrilinéaire est désormais projetée onomastiquement devant le corps social. Parallèlement, le patronyme de la femme mariée signifie et construit pour tous l'alliance matrimoniale dont celle-ci est le vecteur, l'arrachant ainsi aux seuls savoir et mémoire des parentèles impliquées et instituant publiquement l'intégration matrimoniale de la noblesse (au même titre que ce qui semble être l'équivalent structural et pendant consanguin du double patronyme féminin, la mention des quatre aïeuls). Le système anthroponymique qui se met en place à partir de la fin du XVe siècle constitue ainsi un élément essentiel et irremplaçable dans la construction de l'identité sociale du groupement des nobles, à la fois en identifiant les composants du [108] groupe (les lignages, alliés par mariage) et en en construisant la limite sociale (les individus non intégrés matrimonialement, donc non nobles - puisque la noblesse d'un individu découle désormais de son appartenance au groupe noble, du fait qu'il est vom Adel).

Entre les nobles eux-mêmes, la mise en place et la perduration de ce système patronymique contribue à clarifier et hiérarchiser, au niveau du discours, les rapports de parenté, l'appartenance lignagère (la consanguinité) passant avant les liens avec la famille maternelle (l'affinité). Ce faisant, ces procédures de nomination permettent le fonctionnement d'un système matrimonial formellement orienté vers la production et reproduction de «la noblesse » (et non plus seulement/directement la défense et la satisfaction des intérêts patrimoniaux ${ }^{40}$ - lesquelles constituent toutefois l'horizon de la sociogenèse de la noblesse). Parallèlement, la diffusion dans tous les lignages de prénoms non seulement communs mais en outre chevaleresques et spécifiques à la noblesse ne peut qu'avoir accentué une certaine homogénéisation idéologique des nobles, tout en préservant l'identité lignagère.

On peut d'ailleurs même se demander si la diffusion de prénoms spécifiquement nobles et transgressant visiblement les limites discursives des lignages ne constitue pas une manière - là encore discursive - de tempérer les écarts soulignés patronymiquement entre les lignages, en raison de la «ressemblance » (une ressemblance chevaleresque) fondée par l'homonymie, sans parler de l'espèce de proximité (pour ne pas dire d'identité) métaphysique qui peut s'établir entre les membres d'une même famille spirituelle (ayant le même saint patron) ${ }^{41}$, ni même des

\footnotetext{
${ }^{40}$ L'examen des pulsations spatiales du réseau matrimonial des Thüngen montre en effet l'apparition de deux types d'alliances matrimoniales : à côté de celles à dimension locale, orientées selon toute vraisemblance vers l'entretien et/ou la défense des intérêts patrimoniaux, apparaissent au XVe siècle des alliances à plus grande portée, dont les enjeux patrimoniaux sont très douteux, au contraire de leurs enjeux socio-politiques (intégration matrimoniale de la petite aristocratie sur une plus grande échelle, qui correspond exactement à l'espace d'action des nobles franconiens, désigné comme Land zu Franken). Sur l'importance du cadre territorial dans la diffusion des prénoms, voir le cas des Hutten infra (annexe, § III. 2).

${ }^{41}$ C'est le second enseignement à tirer d'un épisode de 1132 rapporté par un continuateur de Sigebert de Gembloux, le chroniqueur Robert du Mont (Monumenta Germaniae Historica - Scriptores, VI, Hannover, 1844, p. 520), selon lequel on fit sortir d'une salle, lors d'un banquet organisé par Henri II Plantagenêt près de Bayeux, tous ceux qui ne portaient pas le prénom Guillaume (qui non vocaretur Willermus). L'épisode montre certes la fréquence du nom (il reste 117 personnes dans la salle en question, sans compter les Guillaume mangeant à la table du roi), comme le remarque Michael MitTERAUER, Ahnen und Heilige. Namengebung in der europäischen Ceschichte, München : C.H. Beck, 1993, p. 243, suivant en cela la logique numérique du chroniqueur (qui tire argument du nombre des Guillaume pour prouver la forte fréquentation du banquet : Et ut appareat multitudo eorum qui interfuerunt...) ; mais il montre
} 
liens de parenté [109] spirituelle fondés sur le baptême. On ne peut en effet exclure que ces prénoms communs aient été en partie diffusés par le biais du parrainage ${ }^{42}$, pratique qui permet de créer ou de renforcer des proximités de type parental entre des personnes entre lesquelles la morale sociale les réprouve ou disqualifie: entre dominants et dominés (vassaux ou dépendants), entre affins et consanguins proches. Le baptême peut être ainsi considéré sous l'angle de la parenté pratique (dont ne doit pas négliger la dimension religieuse) et comme surimposant au discours lignager (tout comme le placement des adolescents nobles comme pages chez leurs oncles maternels 43 ) des relations pratiques adaptées au fonctionnement social.

Les innovations anthroponymiques observés en Franconie à la fin du Moyen Âge sont donc un élément essentiel du succès de la sociogenèse de la noblesse telle qu'on peut l'observer à ce même moment dans cette région. Sachant d'une part qu'il ne peut y avoir de changement social sans changement idéel/discursif (ce qui pose entre autre le problème du statut socioculturel des sources qui font apparaître un tel changement), sachant d'autre part que le changement anthroponymique doit être considéré comme une forme de changement du discours sur la parenté (la différence entre les pratiques textuelles et sigillographiques de nomination montrant combien le changement [110] mesuré doit être rapporté à la spécificité de la source qui l'évoque), on comprend que tout changement social qui passe par une reformulation du discours parental (luimême induit par la prégnance des structures de parenté dans la société considérée) doit s'inscrire dans le champ de l'anthroponymie, non pour s'y refléter mais pour y trouver une de ses formes d'institution les plus essentielles. Les usages anthroponymiques nobles en Franconie à la fin du Moyen Âge apparaissent ainsi comme l'un de ces usages linguistiques essentiels à l'institution sociologique des groupes sociaux. La portée de cet acte d'institution ne peut toutefois être évaluée correctement que si l'on prend en compte l'ensemble des types de sources et l'ensemble des mécanismes sociaux de la société considérée.

également combien l'homonymie pouvait avoir un caractère « rapprochant » : manger ensemble était tout autre chose qu'une action banale (voir par exemple Gerd ALTHOFF, « Der frieden-, bündnis- und gemeinschaftsstiftende Charakter des Mahles im früheren Mittelalter », in : Irmgard BITSCH et alii (dir.), Essen und Trinken in Mittelalter und Neuzeit (Vorträge eines interdisziplinären Symposions, Gießen, 10.-13. Juni 1987), Sigmaringen : Jan Torbecke, (2 éd.) 1990, p. 13-25), cela fonde une familiarité où le seul rapport hiérarchique actif est entre le dispensateur de nourriture et le nourri - mais dans la salle en question, il n'y a que des nourris); en outre, on ne peut exclure (bien que rien dans la source ne permette de l'affirmer), dans cette Normandie du XIIe siècle, que le rassemblement des Guillaume ait eu un objectif spécifiquement mémoriel - commémorer le duc-roi Guillaume le Conquérant -, dont on connaît mieux maintenant la force de construction de groupements sociaux ; enfin, on peut se demander s'il n'existait pas un « effetretour » de l'homonymie sur les homonymes non éponymes (M. MITTERAUER, Ahnen und Heilige..., p. 394, n'envisage un tel « effet-retour » qu'entre l'éponyme et le nommé, effet sur lequel les sources sont muettes) qui ferait entrer tous les homonymes dans une sorte de famille spirituelle polarisée sur le saint ou le prince éponyme.

${ }^{42}$ Les informations à ce sujet sont très partielles (voir infra, annexe, II. 2) et souvent contradictoires (Pierre PÉGEOT, «Un exemple de parenté baptismale à la fin du Moyen Âge. Porrentruy 1482-1500 », in : Les entrées dans la vie. Initiations et apprentissages (XIIe Congrès de la S.H.M.E.S., Nancy, 1981), Nancy : Presses Universitaires de Nancy, 1982, p. 62, observe une transmission des prénoms des parrains/marraines aux filleul(e)s dans $95 \%$ des cas, tandis que les notices concernant les Hohengeroldseck publiées par Ekkehard E. HLAWITSCHKA, "Notizen zur Familiengeschichte der Herren von Hohengeroldseck in der Mitte des 15. Jahrhunderts », Zeitschrift für die Geschichte des Oberrheins, 134, 1986, p. 89-97, ne présentent aucun cas d'éponymie baptismale), donnant ainsi l'impression qu'il n'existait pas de règles générales, mais des pratiques diffusées à des échelles restreintes.

43 Voir le cas de Götz von Berlichingen, qui se retrouve page auprès de son oncle maternel (Helgard ULMSCHNEIDER (éd.), Götz von Berlichingen : Mein Fehd und Handlungen, Sigmaringen : Jan Thorbecke, 1981 (Forschungen aus Württembergisch-Franken, 17), p. 64-74. À noter que la proximité entre les neveux et oncles maternels pourrait avoir été favorisée par le régime démographique propre à la noblesse (voir ma thèse citée supra, note 3, p. 554-555), où l'âge au mariage est plus avancé chez les hommes (vers 30 ans) que chez les femmes (vers 20 ans), si bien que les garçons pouvaient sans doute trouver plus facilement parmi leurs oncles maternels (plus jeunes que leur propre père d'une dizaine d'années) que dans leur famille paternelle des modèles de vitalité chevaleresque (voir le cas de Götz von Berlichingen). 


\title{
La circulation des prénoms dans la petite noblesse franconienne à la fin du Moyen Âge d'après les généalogies des Steckelberg, Hutten, Seinsheim- Schwarzenberg et Thüngen
}

\begin{abstract}
Sources
- Steckelberg : Walther MÖLLER, Stamm-Tafeln westdeutscher Adels-Geschlechter im Mittelalter, N.F., t. 2, Darmstadt: Selbstverlag, 1951, p. 121-123 (planche LXXX);

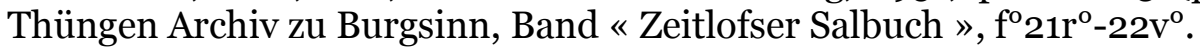

- Hutten : Georg LANDAU, Die hessischen Ritterburgen und ihre Besitzer, t. 3, Kassel, 1836, p. 187-346 ; Walther MöLLER, Stamm-Tafeln westdeutscher Adels-Geschlechter im Mittelalter, N.F., t. 2, Darmstadt : Selbstverlag, 1951, p. 118-121 (planches LXXVIIILXXIX) ; Hans KÖRNER, " Genealogie und Besitz bis zum Ende des Alten Reiches », in : Peter LAUB (dir.), Ulrich von Hutten, Ritter - Humanist - Publizist 1488-1523, Kassel : Hessischer Museumsverband, 1988, p. 57-78; Thüngen Archiv zu Burgsinn, Band «Zeitlofser Salbuch », $\mathrm{f}^{\mathrm{o}} 2 \mathrm{r}^{\mathrm{o}}-24 \mathrm{v}^{\circ}$; Hessisches Staatsarchiv Marburg, K 372.

- Seinsheim : Detlev SCHWENNICKE (dir.), Europäische Stammtafeln. Stammtafeln zur Geschichte der europäischen Staaten, N.F., V, Marburg : J.A. Stargardt, 1988, planches 103-120 ; Karl Fürst zu SCHWARZENBERG, Geschichte des reichsständischen Hauses Schwarzenberg, 2 vol., Neustadt an der Aisch : Degener \& Co, 1968.

- Thüngen : Karl Reinhard Frhr. von THÜNGEN, « Zur Genealogie der Familie derer von Thüngen ", Archiv des Historischen Vereins für Unterfranken und Aschaffenburg, 54, 1912, p. 1-180; Rudolf Frhr. von THÜNGEN, Das reichsritterliche Geschlecht der Freiherrn von Thüngen. Forschungen zu seiner Familiengeschichte (Lutzische Unie), 2 vol., Würzburg, 1926 ; J. MORSEL, Une société politique...
\end{abstract}

Pour des raisons de place, on n'a retenu que les membres masculins (sauf quelques cas de femmes lorsqu'elles servent d'intermédiaires pour la transmission de seigneuries et/ou de prénoms), dont l'ordre d'exposition (de gauche à droite, correspondant théoriquement à l'ordre des naissances) a pu être modifié pour des raisons d'économie graphique 44 . Lorsque l'ordre des naissances est vraiment connu, on en a tenu compte dans le commentaire. Ce faisant, je suis parfaitement conscient de l'aspect relativement fictif et incomplet de la représentation de la parenté fournie par ces généalogies, qui masquent notamment les possibilités de circulation de prénoms par les femmes [112] (épousées ou données en mariage). On doit donc considérer que les généalogies ici présentées ne permettent qu'une première approche du problème de la circulation généalogique des anthroponymes (d'autant que les travaux sur lesquels reposent ces généalogies sont loin d'être de qualité équivalente, tant du point de vue de leur fiabilité que de leur exhaustivité).

\section{Commentaire}

I. La circulation des prénoms au sein même des lignées fait apparaître trois phénomènes (au moins).

I. 1. D'une part, un peut observer, notamment de la fin du XIII' au début du XVe siècle, une alternance de certains prénoms d'une génération à l'autre, avec une relative transmission de prénoms de grand-père à petit-fils ou du moins de génération du grand-père à celle du petit-fils. C'est ainsi le cas de la série Friedrich $\rightarrow$ Frowin $\rightarrow$ Friedrich $\rightarrow$ Frowin chez les Hutten (branche zu Stolzenberg), de la série Erkinger $\rightarrow$

${ }_{44}$ Cf. planches 17 à 19 du fascicule de tableaux généalogiques. 
Ludwig $\rightarrow$ Erkinger $\rightarrow$ Ludwig $(\rightarrow$ ) Erkinger chez les Seinsheim (branche zu Kottenheim aux XIVe-XVI ${ }^{\text {e }}$ siècles), ou encore des séries Marquard $\rightarrow$ Ludwig $\rightarrow$ Marquard $(\rightarrow)$ Ludwig, Dietrich $\rightarrow$ Friedrich $\rightarrow$ Dietrich $\rightarrow$ Friedrich et aussi Andreas $\rightarrow$ Dietrich $\rightarrow$ Andreas $\rightarrow$ Johann $\rightarrow$ Andreas + Eberhard $\rightarrow$ Andreas chez les Thüngen. Toutefois, les sources permettant rarement de connaître avant la fin du XVe siècle l'ordre exact des naissances, il est difficile de préciser si le prénom du grand-père était donné ou non à l'aîné des petits-fils ; surtout, le principe ne semble pas avoir été unanimement respecté, ainsi qu'il apparaît dans un certain nombre de branches où une telle alternance n'est pas observable ; peut-être est-il lié à certains facteurs précis (le lien à un château ou une terre spécifiques).

I. 2. Autre principe inégalement respecté : l'existence de prénoms particulièrement récurrents dans les lignées, où ils sont donnés presque à chaque génération : Ludwig et Friedrich chez les Hutten et les Thüngen, Frowin chez les Hutten, Andreas chez les Thüngen, Erkinger et (aux XIII ${ }^{\mathrm{e}-X I V}$ e siècles) Hildebrand chez les Seinsheim. Toutefois, ces prénoms récurrents spécifient faiblement les topolignées en question: Andreas, Friedrich et Ludwig sont des prénoms courants dans la petite noblesse franconienne 45 ; ce n'est qu'après que les prénoms Friedrich et Ludwig ont cessé d'être importants dans la noblesse (à partir du $\mathrm{XV}^{\mathrm{e}}$ siècle) que leur collation récurrente respectivement chez les Thüngen et chez les Hutten prend une dimension identificatrice. Hildebrand (aux XIII - -XIVe siècles) et Erkinger sont en revanche très fortement liés aux Seinsheim, Frowin l'étant aux Hutten : ils sont presque totalement absents [113] dans d'autres topolignées, où surtout ils ne sont donnés que de manière semble-t-il occasionnelle.

I. 3. Plus probante semble toutefois être la circulation au sein des topolignées de prénoms liés spécifiquement à des châteaux. Ainsi, chez les Hutten, le prénom Ulrich est lié au château du Steckelberg et le prénom Frowin au château du Stolzenberg (Frowin zu Stolzenberg devenu zu Steckelberg n'ayant eu aucun descendant direct portant son prénom) ; ces prénoms spécifiquement « unitopolinéaires » semblent ne se diffuser dans d'autres topolignées qu'à la suite de mariages avec des héritières uniques : Ulrich devient un prénom récurrent dans la branche de Gronau après $1408^{46}-$ date de mariage de Hans zu Gronau et de Margarethe zu Steckelberg -, de même que le prénom Frowin n'est entré dans la branche de Gronau-Steckelberg qu'après le mariage de Friedrich zu Gronau-Steckelberg et de Magdalena zu Stolzenberg en 1473 (le premier fils d'Ulrich zu Gronau-Steckelberg s'étant appelé Ulrich, par référence au Steckelburg47, le second a été nommé Frowin, le troisième prenant le prénom de son grand-père...).

\footnotetext{
45 Pour Ludwig et Friedrich, voir J. MoRSEL, « De l'usage... », tableaux 1,3, 5 ; pour Andreas, on trouve dans les registres de fiefs wurtzbourgeois 1,5 et 11 plusieurs autres topolignées où le prénom est porté.

${ }^{46}$ Le cas d'Ulrich manifeste la difficulté qu'il y a à identifier les déterminants anthroponymiques. En effet, on remarque sur le tableau généalogique la présence d'un Ulrich zu Gronau (1428-1438) beau-frère de Margarethe zu Steckelberg. Deux explications existent : soit le prénom Ulrich est bien " d'origine Steckelberg » et le beau-frère en question a été baptisé ainsi pour manifester la mainmise des Gronau sur le Steckelberg (les dates d'existence connue dudit Ulrich sont tout à fait compatibles avec son éventuelle naissance après 1408); plus vraisemblable toutefois semble être que le prénom Ulrich du beau-frère de Margarethe n'est pas "d'origine Steckelberg », mais plutôt " d'origine Hoelin » : le grand-père maternel de l'Ulrich zu Gronau en question semble en effet s'être nommé Ulrich Hoelin (W. MöLLER, Stamm-Tafeln..., p. 119 ; G. LANDAU, Die hessischen Ritterburgen..., t. 3, p. 305) ; l'apparente continuité onomastique masquerait donc en fait une discontinuité dans la justification des choix.

47 II s'agit de l'enfant terrible de l'Humanisme allemand Ulrich von Hutten, dont les liens avec le Steckelburg (i.e. le château du Steckelberg) ont été rendus célèbres par la fameuse lettre d’Ulrich à l'humaniste nurembergeois Willibald Pirckheimer (dont on trouve une traduction française partielle due à Philippe BRAUNSTEIN dans Georges DUBY (dir.), Histoire de la vie privée, t. 2, Paris : Le Seuil, 1985, p. 531-532) et sont rappelés sur la carte de la Franconie de 1533 due à Sebastian von Rotenhan, qui indique au-dessous du toponyme Steckelperg: Poeta illic Huttenus (voir la reproduction de l'impression réalisée vers 1620 avec les bois d'origine dans Walter M. BROD, « Die Frankenland-Karte des Münchener 'Kunstführers' Peter König, um 1620 », Mainfränkisches Jahrbuch für Geschichte und Kunst, 15, 1963, planche hors-texte).
} 
Chez les Seinsheim, on observe une forte corrélation entre le prénom Erkinger et la seigneurie de Kottenheim : sur 17 porteurs de ce prénom, 9 y sont possessionnés (6 sont explicitement désignés zu Kottenheim et 3 autres non, bien qu'ils y soient possessionnés) ; toutefois, on retrouve ce prénom dans les autres topolignées (zu Wässerndorf, zu Schwarzenberg), si bien qu'on ne peut exclure qu'il ait été considéré comme «transtopolinéaire » et été l'objet de pratiques de nomination visant à rappeler la parenté existant entre toutes les topolignées von Seinsheim (y compris après l'élévation de l'une d'elles au rang baronal en 1429).

[114] Chez les Thüngen, on remarquera notamment l'invention du prénom Reu $\beta$, unique en Franconie, et son attribution pour la première fois au fils d'un des constructeurs du château du Reußenberg ${ }^{48}$, puis à l'un de ses descendants ; le prénom n'a cependant pas été très employé, selon toute vraisemblance parce que le château, au même titre que d'autres (Sodenberg, Thüngen), était tenu en indivision par des membres de toutes les topolignées. La topolignée zu Burgsinn et zu Büchold 49 se caractérise par des prénoms qui lui sont spécifiques, Hildebrand, Balthasar et Sigmund : aucune autre topolignée des Thüngen n’y a recours.

II. Cette circulation topolinéaire de certains prénoms conduit à un deuxième ensemble d'observation, concernant le passage de prénoms d’une lignée à une autre.

II. 1. En effet, si l'on admet que la circulation de certains biens peut commander celle de certains prénoms, on doit alors admettre que ces prénoms peuvent passer d'une lignée à une autre (identifiée chacune par un patronyme spécifique) si tel est le destin des biens en question. Un exemple particulièrement net en est donné par le prénom Ulrich, passé avec l'héritage par les femmes des Steckelberg dans la lignée des Hutten. Il est significatif que ce soit Ulrich et non pas Hermann qui ait été repris : comme le montre la planche Steckelberg-Hutten, Ulrich ne jouissait pas d'une longue tradition chez les Steckelberg, au contraire d'Hermann, que l'on rencontre à plusieurs reprises et dans toutes les branches de la lignée; mais Ulrich I était le premier membre de la branche dont ont fini par hériter les Hutten, celle qui tenait la colline castrale du Steckelberg50. En reprenant le prénom Ulrich, les Hutten se [115] proclamaient ainsi les héritiers non pas des Steckelberg (d'autant qu'une autre branche a encore prospéré durant quelques décennies avant de s'éteindre en ligne masculine à la fin du XIVe siècle, les biens passant en ligne féminine à d'autres lignées), mais de la topolignée zu Steckelberg. Le prénom Ulrich, entré ensuite avec le Steckelberg dans la branche zu Gronau, rend ainsi repérable la succession des héritiers (au gré des partages entre les enfants) du château et des terres dépendantes, c'est-à-dire la topolignée du Steckelberg. On perçoit ainsi deux logiques à l'œuvre, qui se croisent voire se superposent parfois, mais ne sont pas identiques et qu'il faut donc se garder de confondre : une logique

\footnotetext{
${ }^{8}$ D’après la charte par laquelle Andreas, Albrecht, Ludwig et Dietrich von Thüngen reconnaissent en 1333 avoir construit le Reußenberg avec l'accord du comte de Henneberg et le tenir en fief de lui, c'est le comte lui-même qui aurait baptisé le château Reußenberg, situé sur une colline à proximité de Höllrich et dont le nom était (et est toujours) Eichelberg (... gebuwet haben vff dem Eichilberg, der da lyet in der marcke tzü Holderech, ein hus, daz der egenante graff Bertolt, vnser herre, geheyszen hat den Ruzzenberg...) : Georg BRÜcKNER (éd.), Hennebergisches Urkundenbuch, t. 5, Meiningen, 1866, Nr 430. Le prénom Reuß est donc apparu chez les Thüngen après cet acte de construction castrale et onomastique.

${ }^{49}$ Les deux châteaux (allodiaux) de Burgsinn et Büchold ont fait l'objet en 1421 d’une convention de donation réciproque passée entre les cousins germains Sigmund et Balthasar : si l'un vient à mourir sans héritiers directs (le sexe n'est pas précisé !), l'autre ou ses héritiers recevront le château tenu par l'autre (Burgsinn par Sigmund, Büchold par Balthasar), convention validée l'année suivante par le tribunal de cour impérial; la même année (1421), les deux cousins ont également procédé à une donation entre vifs portant sur ces deux châteaux (ces textes sont conservés en copies au Bayerisches Staatsarchiv Würzburg, Archiv Thüngen zu Weißenbach, Bände, Nr 1, fo $40 v^{\circ}-42 r^{\circ} ; \mathrm{Nr}$ 9, p. $10-15$; Akten, $\left.\mathrm{Nr} 2907, \mathrm{f}^{\circ} 1 \mathrm{r}^{\circ}-2 \mathrm{v}^{\circ}\right)$. C'est pourquoi je considère une seule topolignée autour de ces deux châteaux.

$5^{\circ} \mathrm{Au}$ moment de l'héritage, le château est détruit (depuis 1276, sur ordre impérial) et son emplacement (burgstatt) appartient à l'évêque de Würzburg; c'est de ce dernier que Frowin von Hutten reprend en fief, en 1388, le château qu'il a reconstruit en 1387/88. Voir G. LANDAU, Die hessischen Ritterburgen..., t. 3, p. 197-200.
} 
fondée sur l'appropriation et la transmission de la terre (logique topolinéaire) et une logique fondée sur l'appropriation et la succession des enfants dans le cadre de lignées qui s’identifient et se différencient par des patronymes fixes et spécifiques (logique d'affiliation). La non-coïncidence des deux logiques explique d'une part l'ajout au patronyme du complément zu $L^{*}$, d'autre part les efforts visibles réalisés dans le cadre des lignées pour réduire les risques de divergence entre les terres et les enfants, à commencer par les efforts d'exclusion des filles (indemnisées en argent, tout comme d'ailleurs certains cadets sans que cela remette en cause le principe de division successorale : il s'agit simplement de réduire le nombre des diviseurs); ce sont ces efforts qui sont l'objet du discours lignager.

II. 2. Outre cette circulation translinéaire d'origine topolinéaire, on peut également observer des transferts de prénoms d'une lignée à l'autre par les femmes, apparemment non liées à des transferts de biens (à l'exemple de ce qui s'observe peut-être pour le premier Ulrich zu Gronau). Chez les Thüngen, par exemple, il est très vraisemblable que le prénom Hildebrand soit entré par mariage et se soit fixé dans la topolignée zu Burgsinn et Büchold : la mère du premier Hildebrand (et de son frère Wilhelm) est une Gerhaus von Seinsheim ${ }^{1}$ dont la filiation est inconnue, mais se replace certainement dans la partie supérieure gauche de la planche Seinsheim-Schwarzenberg si l'on considère que Hildebrand fonde en 1408 une messe annuelle pour lui, ses enfants et ses ancêtres dans la chartreuse d'Astheim, elle-même fondée par l'Erkinger von Seinsheim élevé en 1429 au rang baronal et qui en a fait la "nécropole » de la branche zu Schwarzenberg52. Ce même prénom Hildebrand, devenu topolinéaire (attaché semblet-il plus spécifiquement au château de Büchold), est passé à son tour par mariage dans la lignée des Steinau-Steinrück, où une fille de Hildebrand zu Büchold, Anna, mariée à Heinrich von Steinau-Steinrück, l'a donné à l'un de ses fils, dont les droits à l'héritage sur le château de Büchold sont devenus évidents à la mort de ses oncles [116] maternels sans héritiers mâles ${ }^{53}$. Dans ce dernier cas (comme dans ceux susmentionnés de l'apparition d'Ulrich dans la branche Hutten zu Gronau et de Frowin dans la branche Hutten zu Gronau-Steckelberg), le port du prénom topolinéaire constituerait une manière de revendication implicite et/ou potentielle de tout ou partie l'héritage du bien en question. Chez les Thüngen, encore, il est également très possible que le prénom Philipp donné à l'aîné des fils de Hildebrand zu Burgsinn, Büchold, Sodenberg et Thüngen ait été donné par référence au beau-père de Hildebrand, Philipp von Hirschhorn, décédé avant le mariage de sa fille Yoland54 ; le prénom semble avoir été en passe de faire souche dans la topolignée de Burgsinn, puisqu'il réapparaît chez le petitfils du Philipp von Thüngen en question.

Outre ce transfert de prénoms dans le cadre de mariages, on ne peut exclure un transfert dans le cadre de parrainages, sur lequel je n'ai malheureusement, pour les lignées ici choisies, pour ainsi dire aucune donnée. Le seul cas documenté est celui de Wilhelm von Thüngen, fils d'Albrecht zum Sodenberg, Thüngen et Windsheim et né en 1508 ou 1509: on sait que son parrain fut Wilhelm von Finsterlohe55. Mais Wilhelm n'est ni un prénom nouveau chez les Thüngen (un oncle paternel d'Albrecht s'appelle ainsi), ni dans la noblesse franconienne au début du XVI ${ }^{\mathrm{e}}$ siècle ${ }^{56}$ : il existe donc bien d'autres déterminismes possibles que l'homonymie baptismale. Si c'était effectivement

\footnotetext{
${ }^{51}$ Voir supra, note 13.

${ }^{2}$ Fondation par Hildebrand : notice conservée au Bayerisches Staatsarchiv Würzburg, Archiv Thüngen zu Weißenbach, Bände, $\mathrm{Nr} 1, \mathrm{f}^{\mathrm{o}} 32 \mathrm{v}^{\mathrm{o}}$; sur Astheim, voir N. BACKMUND, Die kleineren Orden..., p. 59-60, et Detlev SCHWENNICKE, Europäische Stammtafeln..., planche 104.

53 Voir par exemple Bayerisches Staatsarchiv Würzburg, Archiv Thüngen zu Weißenbach, Urkunden, Nr 589.

54 Voir le contrat de mariage conservé en copies au Bayerisches Staatsarchiv Würzburg, Archiv Thüngen zu Weißenbach, Bände, $\mathrm{Nr} 1, \mathrm{f}^{\circ} 49 \mathrm{r}^{\circ}-5 \mathrm{Or}^{\circ}$, et au Bayerisches Staatsarchiv Bamberg, A 205, Lade 732, Nr 2309, et édité dans ma thèse mentionnée supra, note 3 .

55 Julius-Spitalsarchiv zu Würzburg, Literalien, $\mathrm{Nr}$ 105, p. 287.

${ }_{56}^{6}$ Voir supra, tableau 1.
} 
le cas ici, nous resterions toutefois dans un contexte fortement déterminé par les alliances matrimoniales : Wilhelm von Finsterlohe était en effet le fils d'une Ursula von Thüngen et parent de son filleul au troisième degré canon (illustrant ainsi l'hypothèse d'A. Guerreau-Jalabert d'un « choix probable d'une bonne partie des parents spirituels à l'intérieur du très vaste cercle des parents déjà interdits pour consanguinité ou affinité $\gg 57)$.

Que ce soit par héritage, par mariage, ou par parrainage, ce transfert de prénoms d'une lignée à une autre, qui se surimpose à une circulation plus proprement linéaire (dans le cadre de la lignée - qu'il s'agisse d'une seule topolignée ou d'un ensemble de topolignées $5^{8}$ ) et pouvant être insérée dans une [117] logique discursive lignagère, illustre clairement que les prénoms ne circulent pas de manière rigoureusement patrilinéaire/lignagère, un système patrilinéaire excluant tout autant la transmission des biens par les filles que la transmission des noms.

III. À ces deux formes de transmission parentale des prénoms (intralinéaire/lignagère et extralinéaire i.e. topolinéaire et/ou parentélaire) semble s'en ajouter une troisième, qui prendrait pour cadre des ensembles plus vastes que les simples lignées et parentèles.

III. 1. Un premier cadre semble être celui de la chrétienté : on observe en effet dans les trois lignées (Hutten, Seinsheim et Thüngen) l'apparition du prénom Johann (le plus souvent sous sa forme hypocoristique Hans) dans la seconde moitié du XIV siècle, ce qui correspond à un phénomène général en Franconie à cette époque (avec le décalage temporel des nobles par rapport aux non-nobles). Il se maintient bien dans ces trois lignées 59 au moins durant le XVe siècle, mais disparaît chez les Thüngen au XVIe siècle. On observera dans les trois lignées l'apparition concomitante de Melchior (vers 1500), auquel répond peut-être chez les Thüngen Kaspar (prénom donné pour la première fois au frère de Melchior) mais sans doute pas Balthasar (prénom «topolinéaire » à Büchold).

III. 2. Un second cadre semble être la Ritterschaft franconienne. On remarque en effet chez les Seinsheim-Schwarzenberg comme chez les Thüngen l'apparition, à partir de la génération ${ }^{60}$ née dans la première moitié $d u \mathrm{XV}^{\mathrm{e}}$ siècle, de prénoms de saints chevaliers

\footnotetext{
57 « Prohibitions canoniques... », p. 303.

${ }_{58}^{8} \mathrm{La}$ particule $z u$ peut servir selon les cas à identifier une topolignée (notamment lorsque le patronyme n'est lui-même pas un toponyme, par exemple dans le cas des Truchseß zu Wetzhausen, Truchse $\beta$ signifiant "écuyer tranchant », dapifer, et renvoyant à une fonction aulique devenue héréditaire lorsque la lignée n'était encore que ministériale ; il est d'ailleurs significatif que le nom ait été parfois écrit Truchseß von Wetzhausen, comme si le remplacement de particule devait exprimer la dimension linéaire et non pas seulement topolinéaire de la lignée : voir par exemple Bayerisches Staatsarchiv Würzburg, Archiv Thüngen zu Weißenbach, Akten, Nr 2907, $\mathrm{f}^{\circ}{ }^{\circ} \mathrm{r}^{\circ}$ ) ou à distinguer des topolignées au sein d'une lignée. Seul l'examen des terres et châteaux détenus, ainsi que d'une éventuelle politique de bouclage consanguin « agnatique », permet de savoir s’il s'agit de topolignées se reconnaissant d'une même lignée et susceptibles de s'entreléguer les biens auxquels elles s’identifient, ou alors s’il s'agit de lignées distinctes centrées sur des biens spécifiques.

59 Chez les Thüngen, on trouve au XVe siècle au moins deux autres Hans qui ne figurent pas sur le tableau ici fourni en raison de leur filiation inconnue; voir Rudolf Frhr. von THÜNGEN, Das reichsritterliche Geschlecht..., planches généalogiques.

6o La notion de génération est problématique, car elle se réfère à deux critères distincts : la date de naissance (d'où l'expression par exemple de "génération de l'après-guerre ») et la position généalogique (génération des parents, génération des enfants). Or, les tableaux généalogiques ne font apparaître que les positions généalogiques, faisant appartenir à une même génération des individus d’âges très différents (avec des écarts de l'ordre d'une génération : 30 ans si l'on considère l'âge au mariage courant chez les hommes notamment entre la gauche et la droite du tableau, où les écarts résultant de la présentation de gauche à droite de l'ordre des naissances finissent par se cumuler). Chez les Thüngen, par exemple, on fera difficilement appartenir à la même génération, bien qu'ils figurent sur la même ligne, Georg zu Reußenberg, Sodenberg, Thüngen und Lauda, fils aîné d'un second fils d'un fils unique d'un fils aîné, etc. et représenté à gauche du tableau, et Eustachius, Konrad ou Bernhard, fils d'un fils unique d'un fils cadet d'un fils unique d'un fils cadet d'un fils cadet, etc. et représentés à droite du tableau. Je n'emploie donc ici le terme qu'à des fins de repérage dans les tableaux généalogiques.
} 
totalement absents jusqu'alors : Georg [118] (trois occurrences - autant que Johann et Erkinger - chez les Seinsheim vers 1450, une occurrence dans la génération suivante, deux $^{61}$ à la dernière représentée ; une occurrence chez les Thüngen vers 1450, une à la génération suivante et deux à la dernière génération représentée); Martin (deux occurrences chez les Seinsheim vers 1450, une autre à la génération suivante ;

une occurrence à la dernière génération représentée chez les Thüngen); Sebastian (une occurrence chez les Seinsheim); Moritz, Eustachius, Pankraz (une occurrence de chaque chez les Thüngen), etc. La fortune de Wilhelm (présent chez les Seinsheim et les Thüngen depuis la fin du XIVe siècle et dans presque toutes les générations suivantes ${ }^{62}$ ) est difficile à interpréter, un prénom traditionnel pouvant être réactivé par une dimension plus spécifiquement chevaleresque ${ }^{63}$. Il en va d'ailleurs de même pour Michael, présent chez les Seinsheim depuis la fin du XIVe siècle. Le succès de Philipp (chez les Seinsheim et les Thüngen depuis le milieu du XVe siècle, chez les Hutten de Franconie depuis la fin du $\mathrm{XV}^{\mathrm{e}}$ siècle) est également problématique: l'aspect déterminant est-il l'apôtre, le tueur de dragon, le père d'Alexandre (l'un des neuf Preux), " celui qui aime les chevaux » 64 ? Ces deux dernières éventualités en feraient un prénom relevant spécifiquement de l'univers chevaleresque.

On remarque aisément que ces prénoms ne sont pas largement dominants dans les générations où ils apparaissent, sans parler de leur absence totale chez les Hutten. Ces prénoms, dont j'ai souligné l'importance statistique notable, sont donc au mieux les plus communs d'une lignée à l'autre, et non pas les préférés des lignées. L'absence de ces prénoms chez les Hutten ainsi d'ailleurs que la notable résistance de prénoms qui déclinent dans les autres lignées, notamment Konrad (dans une topolignée non représentée ici) s'expliquent sans doute pour partie par le fait que les Hutten n’appartiennent [119] que marginalement à la Ritterschaft franconienne : ils sont à

${ }^{61}$ Chez les Seinsheim-Schwarzenberg comme chez les Thüngen, on peut observer au début du XVIe siècle une nouveauté : les prénoms doubles (Wilhelm-Balthasar, Georg-Friedrich, Hans-Ulrich, Hans-Christoph, OttoHeinrich, Georg-Ludwig, Hans-Jörg). Estimant qu'il s'agit là d'une double référence, du choix d'un double patronage, j'ai pris le parti de décomposer les prénoms doubles en deux prénoms et de considérer chacun d'eux séparément lors des comptages.

${ }^{62}$ Chez les Thüngen, on trouve au $\mathrm{XV}^{\mathrm{e}}$ siècle au moins deux autres Wilhelm qui ne figurent pas sur le tableau ici fourni en raison de leur filiation inconnue; voir Rudolf Frhr. von THÜNGEN, Das reichsritterliche Geschlecht..., planches généalogiques.

${ }_{63}$ H. KRUSE et alii, Ritterorden und Adelsgesellschaften..., p. 8-9 et 27, montrent que saint Guillaume (= de Gellone, popularisé par la chanson de geste Willehalm) est un patron fréquent, associé dans un cas à saint Georges, de sociétés de chevalerie dans le Sud-Ouest de l'Empire (c'est-à-dire dans ces régions dont l'évolution socio-politique m'intéresse ici).

${ }^{64}$ Rappelons la place tout à fait exceptionnelle dont jouit le cheval dans la mentalité " chevaleresque », place qui reste cependant encore mal connue en dehors de son importance globale. Il ne me paraît donc pas du tout inconcevable que la connotation hippophile du prénom Philipp ait pu être déterminante dans la diffusion de ce prénom chez des nobles attachés au modèle chevaleresque, tandis qu'il reste très peu représenté chez les non-nobles (2 occurrences sur 3645 en 1467-1476, 2 sur 246 en 1500-1550). 
cheval sur la Hesse et la Franconie et se rattachent politiquement (c'est-à-dire par leurs alliances militaires et matrimoniales) tout autant aux nobles de l'une que de l'autre région: c'est peut-être dans ce contexte que l'on peut expliquer l'apparition des prénoms Georg et Moritz à la fin du XVe siècle dans la topolignée plus nettement franconienne (zu Arnstein puis zu Frankenberg) des Hutten (non représentée ici65). Le cas des Hutten illustre ainsi clairement combien les études de transmission des prénoms doivent prendre en compte les dimensions régionales/locales de collation de ceux-ci. L'étude de la prénomination en Souabe, par exemple, autre lieu d'intense activité de mobilisation nobiliaire, pourrait ainsi faire apparaître des résultats très différents. Le modèle franconien de formalisation anthroponymique du groupe nobiliaire se construisant en "noblesse » n'est ainsi pas nécessairement le modèle nobiliaire : il est le résultat de la convergence de tensions structurelles et de conditions locales.

${ }_{65}$ Voir H. KÖRNER, « Genealogie und Besitz... », p. 63-69 et planches 4-5; W. MÖLLER, Stamm-Tafeln..., p. 121 et planche LXXVIII, nomme d'ailleurs cette branche fränkische Linie. 
Steckelberg

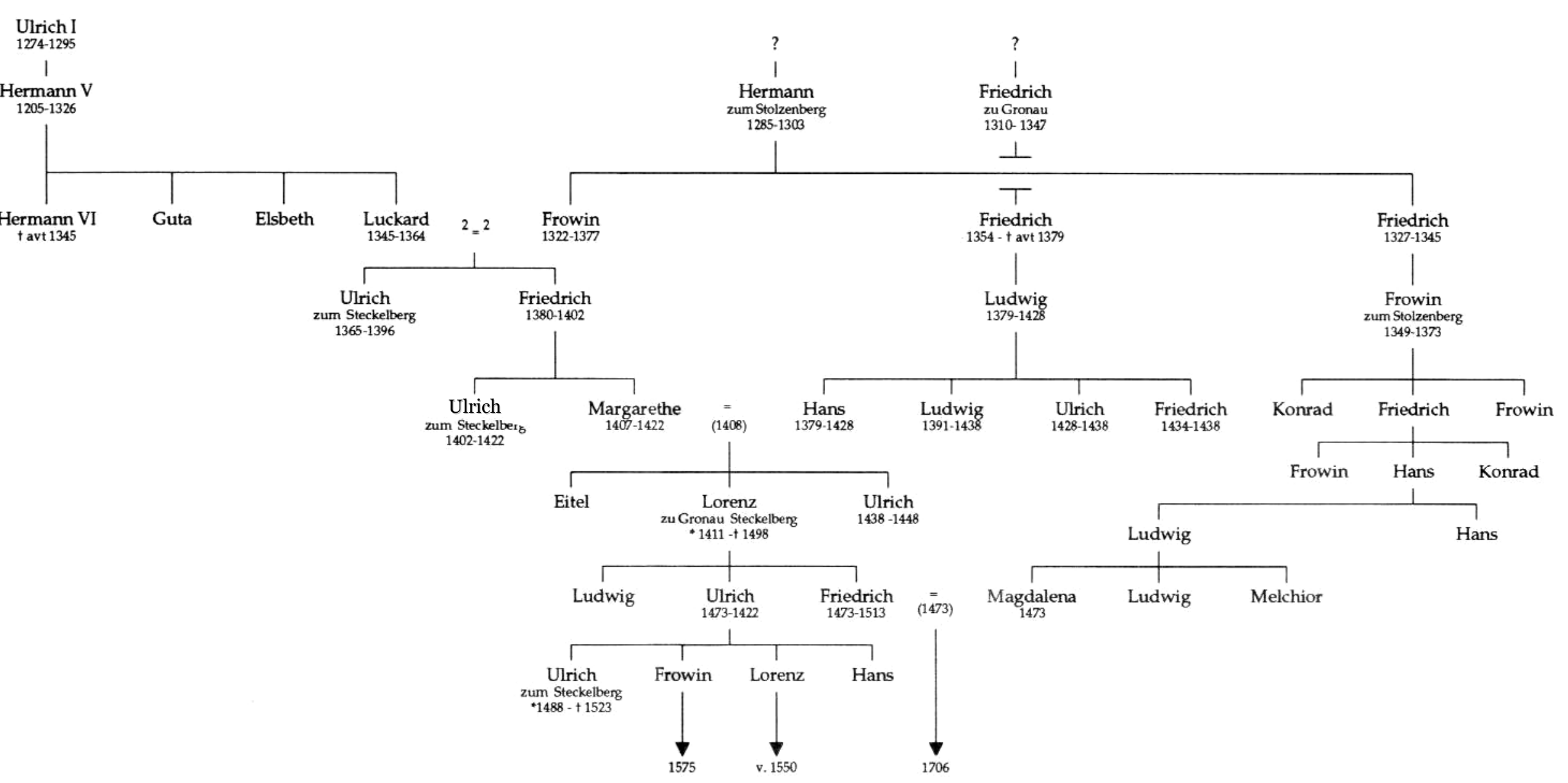

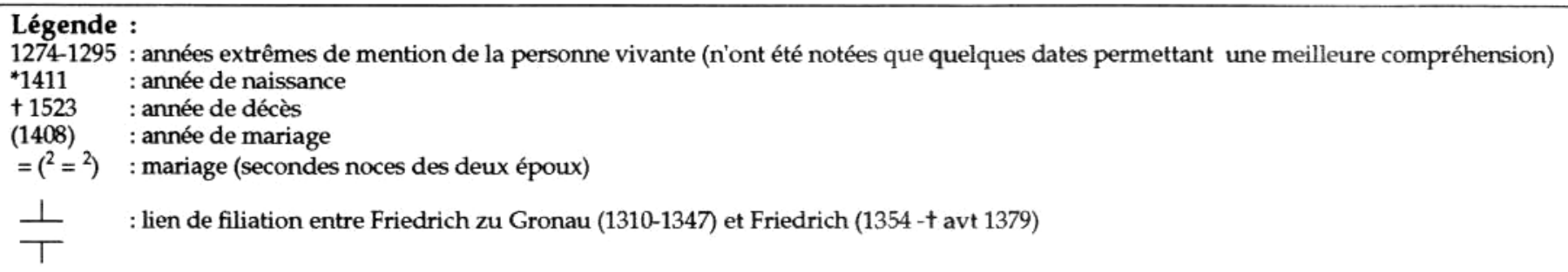




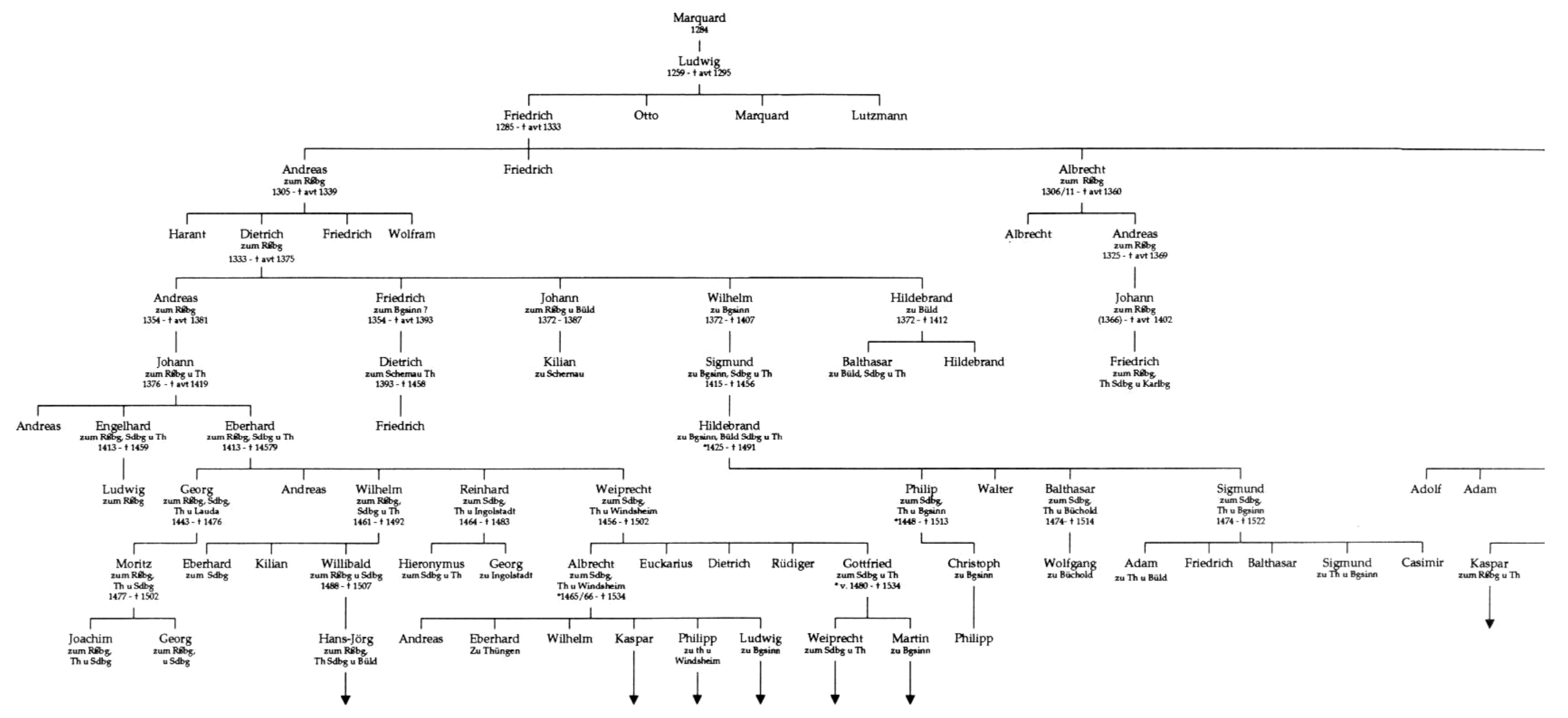

\begin{tabular}{|c|c|}
\hline $\begin{array}{l}\text { Légende } \\
1372-1387 \\
+1425 \\
+1407 \\
(1366) \\
\downarrow\end{array}$ & $\begin{array}{l}: \\
\text { années extrêmes de mention de la personne vivante (n'ont été notées que les dates des Thüngen ayant eu des fils) } \\
\text { année de naissance } \\
: \text { année de decés } \\
\text { mention incertaine } \\
\text { prolongemements en ligne masculine à l'époque modeme }\end{array}$ \\
\hline $\begin{array}{l}\text { R\&bg } \\
\text { Sdbg } \\
\text { Th } \\
\text { Bgsinn } \\
\text { Buld } \\
\text { Karlbg }\end{array}$ & $\begin{array}{l}\text { : ReuRenberg } \\
\text { : Sodenberg } \\
\text { : Thüngen } \\
\text { : Burgsinn } \\
\text { : Karchold } \\
\text { Karlburg }\end{array}$ \\
\hline
\end{tabular}




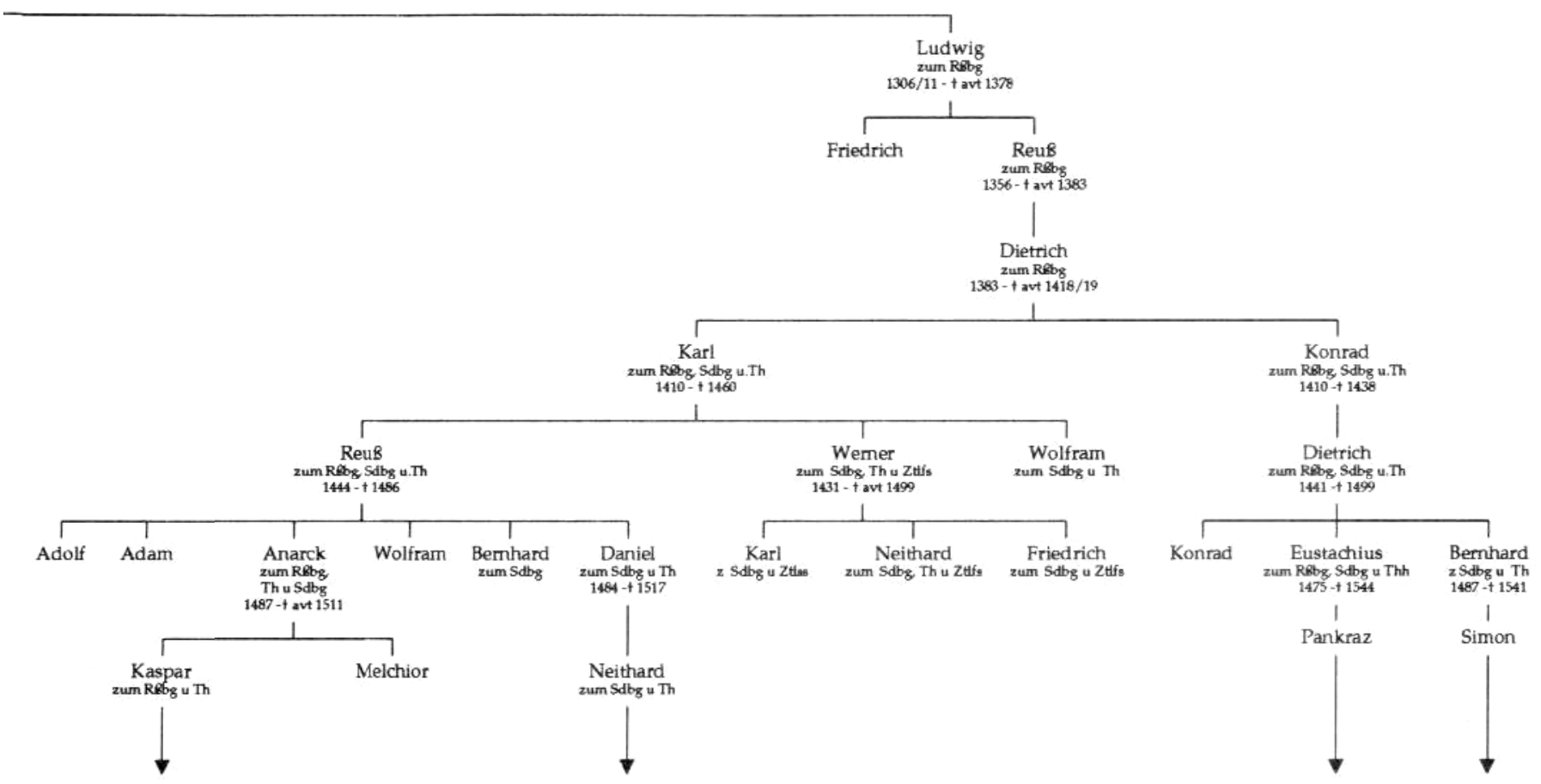




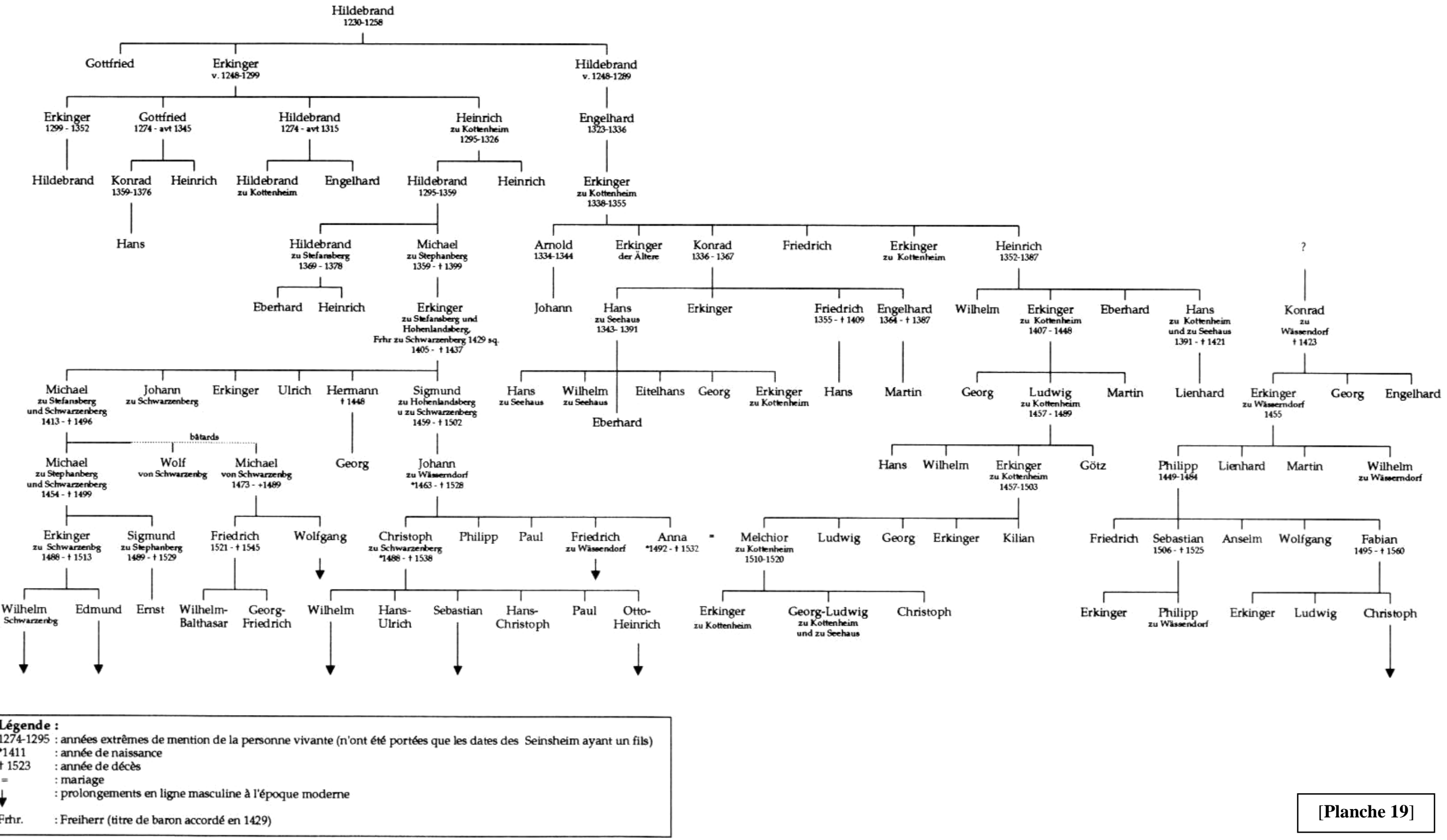

